\title{
Relativity: Towards a New Interpretation
}

\section{Carmine Cataldo}

Independent Researcher, Battipaglia, Italy

Email: catcataldo@hotmail.it

How to cite this paper: Cataldo, C. (2019) Relativity: Towards a New Interpretation. Journal of High Energy Physics, Gravitation and Cosmology, 5, 790-849. https://doi.org/10.4236/jhepgc.2019.53041

Received: May 21, 2019

Accepted: July 7, 2019

Published: July 10, 2019

Copyright $\odot 2019$ by author(s) and Scientific Research Publishing Inc. This work is licensed under the Creative Commons Attribution International License (CC BY 4.0).

http://creativecommons.org/licenses/by/4.0/

\begin{abstract}
The aim of this paper essentially lies in attributing a new meaning, coherent with phenomenological reality, to several phenomena usually classified as relativistic, such as the alleged increase of the mean lifetime of muons and the gravitational redshift. According to the model herein proposed, all the relativistic equations preserve their validity, albeit with a different connotation. We consider a Simple-Harmonically Oscillating Universe, characterized by a null curvature parameter, postulating the existence of a further spatial dimension, not directly perceivable. Time is considered as being absolute, although instruments and devices of whatever kind, commonly employed to measure it, may be significantly influenced by motion and gravity. The Planck Constant is regarded as a parameter, locally variable and subjected to a cyclic evolution. Time and space are treated as quantized physical quantities.
\end{abstract}

\section{Keywords}

Special Relativity, Lorentz Transformations, General Relativity, Cosmological Models, Black Holes, Schwarzschild Solution, Variable Planck Constant

\section{Introduction}

The paper consists, net of this short introduction, of 9 Sections or Paragraphs.

In Paragraph 2, the well-known compatibility between General Relativity (from now onwards GR) and a Simple-Harmonically Oscillating Universe, flat and conventionally singular at $t=0$, is accurately discussed: in detail, starting from the first Friedmann-Lemaitre Equation, we carry out a step-by-step derivation of the simple equation that describes the cyclic variation (over time) of the Scale Parameter (the radius, in our case).

In Paragraph 3, the existence of a further spatial dimension is postulated. The Universe is identified with a 4-Ball, the radius of which may evolve in accordance with the relation derived in the previous paragraph, and the concept of material point is definitively replaced by the one of material segment. In the light 
of these assumptions, we firstly carry out an alternative deduction of the so-called Mass-Energy Equivalence, albeit with a different meaning: subsequently, by introducing a non-material term (which may be related to the so-called Quantum Potential), we obtain a rewriting of the Conservation of Energy. Finally, exploiting an opportune space quantization, we achieve the expression for so-called Relativistic Energy, the connotation of which, coherently with the aim of this paper, turns out to be undoubtedly distant from the norm.

In Paragraph 4, we address the Lorentz Transformations, backbone of Special Relativity (from now onwards SR). At this stage, we introduce more explicitly the absoluteness of time. In the light of this fundamental assumption, undeniably tough, we carry out, by exploiting the outcomes attained in the previous paragraph, an unconventional derivation of the Lorentz Transformations, both in direct and inverse form. Obviously, the equations we obtain, although formally coinciding with the Lorentz Transformations, can no longer be considered as being relativistic (at least in the Einsteinian sense of the term) and acquire a meaning concretely different from the one usually ascribed to them.

In Paragraph 5, in order to provide a better understanding of some noteworthy positions carried out in the second paragraph, we propose an alternative deduction of the Friedmann-Lemaitre Equations. At the end of the section, however, the Metric Expansion/Contraction is manifestly and uncompromisingly considered as being an apparent phenomenon, related to the variability of the Planck Constant. In detail, by resorting to the Generalized Uncertainty Principle, we improve the quantization introduced in the second paragraph, so attaining the writing of the first Friedmann-Lemaitre equation as a function of a timedependent Planck “Constant”.

In Paragraph 6, we start dealing with Gravitation. Firstly, taking into account the results obtained in the previous paragraph, we definitively postulate a static scenario. Secondly, in order to ideally create Gravitational Singularities, we build a simple model entirely based on the redistribution of mass, the total amount of which is considered as being constant. By means of the above-mentioned model, we determine how space fabric is deformed by mass. Finally, we check the compatibility between the hypothesized scenario and the quantization introduced in the second paragraph (and improved in the fifth).

In Paragraph 7, the problem of Black Holes is addressed. According to our theory, in order to obtain something similar to a real (non-rotating and noncharged) Black Hole, all the available mass should be concentrated in a single point. By resorting to the model introduced in the previous paragraph, we define a Pseudo-Newtonian Gravitational Potential, and discuss the motion of a FreeFalling Particle. In this section, we start to abandon the idea of warped space, replacing it with the idea of variable space (and time) quanta: in other terms, we postulate a local variability of the Planck Constant. At the end of the paragraph, we carry out a parameterization, which formally involves also the quantization, by means of which we will be able to derive a Schwarzschild-Like Solution. 
In Paragraph 8, we examine the well-known Schwarzschild Solution. Starting from a flat metric, obtained by exploiting the parameterization introduced in the previous paragraph, we initially carry out, by resorting to a preliminary Hilbert-Like Approach, a conventional derivation of a Schwarzschild-Like Metric. Next, starting from the same initial flat metric, but following a completely different line of reasoning, we deduce an infinite class of Schwarzschild-Like Solutions (including the metrics derived by Droste and Brillouin). At the end of the section, we briefly address the Gravitational Redshift, regarded as exclusively related to the local variability of the Planck Constant.

In Paragraph 9, in the light of the image of the alleged Black Hole, placed at the heart of M87, recently captured by the Event Horizon Telescope, we initially provide a short outline of an interesting theory, fully coherent with GR, according to which the above-mentioned image may portray a so-called Eternally Collapsing Object (and not a Black Hole). Next, by introducing the concept of velocity as a complex vector, we propose a further explanation (non-relativistic, unlike the previous), compatible with the image in question, which may peremptorily exclude the existence of Black Holes (and Event Horizons).

\section{The Oscillating Universe in General Relativity}

According to Harrison's classification [1], there are four main groups of Uniform Cosmological Models compatible with GR: static, asymptotic, monotonic, and oscillatory.

Each of the above mentioned groups, in turn, may be subdivided into sub-groups or classes. We herein exclusively address the upper-bounded oscillatory class characterized by a flat (Euclidean) geometry: in particular, we will derive a Simple-Harmonically Oscillating Model, conventionally singular at $t=0$.

\subsection{Oscillatory Class with $k=0$}

For a uniform Universe, with the usual hypotheses of homogeneity and isotropy, we can write the first Friedmann-Lemaittre Equation [2] [3] as follows:

$$
\left(\frac{\mathrm{d} R}{\mathrm{~d} t}\right)^{2}=\frac{1}{3}\left(8 \pi G \rho+\Lambda c^{2}\right) R^{2}-k c^{2} .
$$

$R$ represents the Scale Factor [3], $G$ the Gravitational Constant, $\rho$ the Density, $\Lambda$ the so-called Cosmological Constant [3], $k$ the Curvature Parameter [3], the value of which depends on the hypothesized geometry, and $c$ the Speed of Light.

As is well known, if we denote with $E$ Energy, with $T$ the Thermodynamic Temperature, with $S$ the Entropy, with $p$ the Pressure, and with $V$ the Volume, we can write:

$$
\mathrm{d} E=T \mathrm{~d} S-p \mathrm{~d} V .
$$

If we identify the evolution of the Universe with an isentropic process, from the previous Equations we obtain:

$$
\mathrm{d} E+p \mathrm{~d} V=0 .
$$


According to Mass-Energy Equivalence [3] [4], we have:

$$
E=M c^{2} \text {. }
$$

Obviously, we can write:

$$
\frac{\mathrm{d} M}{\mathrm{~d} t}=\frac{\mathrm{d}}{\mathrm{d} t}(\rho V)=V \frac{\mathrm{d} \rho}{\mathrm{d} t}+\rho \frac{\mathrm{d} V}{\mathrm{~d} t} .
$$

Taking into account Equations (2.4) and (2.5), from Equation (2.3) we obtain:

$$
\begin{gathered}
c^{2} V \frac{\mathrm{d} \rho}{\mathrm{d} t}+c^{2} \rho \frac{\mathrm{d} V}{\mathrm{~d} t}+p \frac{\mathrm{d} V}{\mathrm{~d} t}=0, \\
V \frac{\mathrm{d} \rho}{\mathrm{d} t}+\left(\rho+\frac{p}{c^{2}}\right) \frac{\mathrm{d} V}{\mathrm{~d} t}=0 .
\end{gathered}
$$

Since we consider $V \propto R^{3}$, with $R \neq 0$, we have:

$$
\frac{1}{V} \frac{\mathrm{d} V}{\mathrm{~d} t}\left(\rho+\frac{p}{c^{2}}\right)=\frac{3}{R} \frac{\mathrm{d} R}{\mathrm{~d} t}\left(\rho+\frac{p}{c^{2}}\right) .
$$

From the last two Equations, we immediately deduce the so-called Fluid Equation:

$$
\dot{\rho}=\frac{\mathrm{d} \rho}{\mathrm{d} t}=-\frac{3}{R} \frac{\mathrm{d} R}{\mathrm{~d} t}\left(\rho+\frac{p}{c^{2}}\right)=-3 \frac{\dot{R}}{R}\left(\rho+\frac{p}{c^{2}}\right) .
$$

According to Zeldovich [5], the relation between pressure and density (the Equation of State) can be written as follows:

$$
p=(v-1) \rho c^{2} \text {. }
$$

Evidently, the Universe is identified with a barotropic fluid (pressure exclusively depends on density). The value of $v$, hypothesized as being constant, solely depends on the type of fluid we take into consideration (matter, radiation, relativistic gas, dark energy, etc.): the general accepted values lie in the range $1 \leq v \leq 4 / 3 \quad[5]$.

From Equation (2.8), taking into account Equation (2.10), we obtain:

$$
\frac{\mathrm{d} \rho}{\rho}=-3 v \frac{\mathrm{d} R}{R} \text {. }
$$

As a consequence, if we denote with $C$ the constant of integration, we can easily deduce the following:

$$
\rho R^{3 v}=C
$$

Equation (2.1) can be evidently rewritten as follows:

$$
\left(\frac{\mathrm{d} R}{\mathrm{~d} t}\right)^{2}=\frac{8 \pi G \rho R^{3 v}}{3} R^{2-3 v}+\frac{1}{3} \Lambda c^{2} R^{2}-k c^{2} .
$$

By virtue of Equation (2.12), the underlying new constant can be now defined:

$$
C_{v}=\frac{8 \pi G \rho R^{3 v}}{3}=\frac{8 \pi G C}{3} \text {. }
$$

By substituting the previous identity into Equation (2.13), we obtain: 


$$
\dot{R}^{2}=C_{v} R^{2-3 v}+\frac{1}{3} \Lambda c^{2} R^{2}-k c^{2} .
$$

Denoting with $\omega$ the Pulsation of the Oscillating Universe we want to describe, we can carry out the following position involving the cosmological constant:

$$
\Lambda=-3\left(\frac{\omega}{c}\right)^{2}
$$

If we set $k=0$, by substituting Equation (2.16) in Equation (2.15), we finally obtain:

$$
\dot{R}^{2}=C_{v} R^{2-3 v}-\omega^{2} R^{2} .
$$

From the previous equation, we can deduce as follows:

$$
\begin{gathered}
\frac{\mathrm{d} R}{\mathrm{~d} t}=\sqrt{C_{v}} R^{1-\frac{3}{2} v} \sqrt{1-\left(\frac{\omega R^{\frac{3}{2}}}{\sqrt{C_{v}}}\right)^{2}}, \\
\sqrt{C_{v}} R^{1-\frac{3}{2} v} \sqrt{\frac{\mathrm{d} R}{1-\left(\frac{\omega R^{\frac{3}{2}}}{\sqrt{C_{v}}}\right)^{2}}}=\mathrm{d} t, \\
\frac{\mathrm{d}\left(\frac{\omega R^{\frac{3}{2} v}}{\left.\sqrt{C_{v}}\right)^{2}}\right.}{\sqrt{1-\left(\frac{\omega R^{\frac{3}{2}}}{\sqrt{C_{v}}}\right)^{2}}}=\frac{3}{2} \mathrm{~d} t .
\end{gathered}
$$

If we impose that the radius of curvature assumes a null value when $t=0$, from the prior equation we can deduce:

$$
\begin{gathered}
\arcsin \left(\frac{\omega R^{\frac{3}{2} v}}{\sqrt{C_{v}}}\right)=\frac{3}{2} v \omega t, \\
R^{3 v}=\frac{C_{v}}{\omega^{2}} \sin ^{2}\left(\frac{3}{2} v \omega t\right)=\frac{C_{v}}{2 \omega^{2}}[1-\cos (3 v \omega t)], \\
R=\left(\frac{C_{v}}{2 \omega^{2}}\right)^{\frac{1}{3 v}}[1-\cos (3 v \omega t)]^{\frac{1}{3 v}} .
\end{gathered}
$$

According to Equation (2.23), we have formally achieved a model of Universe belonging to the oscillatory class ("O Type" in Harrison's Classification) [1].

From Equations (2.14) and (2.22), we immediately obtain:

$$
\rho=\frac{3}{8 \pi G} \frac{C_{v}}{R^{3 v}}=\frac{3 \omega^{2}}{4 \pi G} \frac{1}{1-\cos (3 v \omega t)} .
$$


Finally, by taking into account Equation (2.16), we can write the foregoing as follows:

$$
\rho=-\frac{\Lambda c^{2}}{4 \pi G} \frac{1}{1-\cos (3 v \omega t)}
$$

\subsection{A Simple-Harmonically Oscillating Universe}

If we set $v=1 / 3$, from Equation (2.23) we obtain:

$$
R=\frac{C_{1 / 3}}{2 \omega^{2}}[1-\cos (\omega t)] .
$$

In other terms, we have found a simple-harmonically oscillating universe characterized by a variable density whose value, taking into account Equation (2.25), is provided by the following relation:

$$
\rho=-\frac{\Lambda c^{2}}{4 \pi G} \frac{1}{1-\cos (\omega t)} .
$$

Denoting with $A$ the Amplitude of the motion, taking into account Equation (2.26), we can immediately write:

$$
A=\frac{C_{1 / 3}}{2 \omega^{2}} .
$$

If we set $\omega t=\pi / 2$, denoting with $R_{m}$ the mean radius, from Equations (2.26) and (2.28) we have:

$$
R\left(\frac{\pi}{2}\right)=A=R_{m} .
$$

By virtue of the previous, from Equation (2.27) we have:

$$
\rho_{m}=\rho\left(R_{m}\right)=-\frac{\Lambda c^{2}}{4 \pi G} .
$$

Evidently, taking into account Equations (2.28) and (2.29), Equation (2.26) acquires the following banal form:

$$
R=R_{m}[1-\cos (\omega t)]
$$

Now, from Equation (2.12), since we have set $v=1 / 3$, we can write:

$$
\rho R=\rho_{m} R_{m} .
$$

From Equations (2.14), (2.28) and (2.32) we have:

$$
\begin{gathered}
\omega^{2}=\frac{C_{1 / 3}}{2 R_{m}}=\frac{4 \pi G}{3} \rho \frac{R}{R_{m}}=\frac{4 \pi G \rho_{m}}{3}, \\
\left(\omega R_{m}\right)^{2}=\frac{2\left(\frac{2}{3} \pi R_{m}^{3} \rho_{m}\right) G}{R_{m}} .
\end{gathered}
$$

\subsection{Particular Case: Further Positions}

We can now carry out the following positions [5]: 


$$
\begin{gathered}
M_{m}=\frac{2}{3} \pi R_{m}^{3} \rho_{m}, \\
\omega R_{m}=c .
\end{gathered}
$$

The position in Equation (2.35), at a first glance undoubtedly puzzling, will be at a later time easily understood when dealing with the concept of "Global Central Symmetry".

From Equation (2.34), taking into account Equations (2.35) and (2.36), denoting with $R_{s}$ the so-called Schwarzschild Radius [3] [7], we can write:

$$
R_{m}=\frac{2 M_{m} G}{c^{2}}=R_{s}\left(M_{m}\right) \text {. }
$$

In the light of the outcomes so far achieved, we can evidently write the following:

$$
\begin{gathered}
\omega t=\frac{c t}{R_{m}}=\alpha, \\
R=R_{m}(1-\cos \alpha), \\
\cos \alpha=1-\frac{R}{R_{m}}, \\
\dot{R}=\frac{\mathrm{d} R}{\mathrm{~d} t}=c \sin \alpha, \\
\ddot{R}=\frac{\mathrm{d} \dot{R}}{\mathrm{~d} t}=c \omega \cos \alpha=\frac{c^{2}}{R_{m}}\left(1-\frac{R}{R_{m}}\right) .
\end{gathered}
$$

The beginning of a new cycle $(t=0)$ occurs when the radius of curvature assumes a null value. The problem related to the singularity [1] [8], herein not concretely addressed, may be solved by resorting to a quantization (see paragraph): in other terms, we should imagine a "Quantum Leap" (actually, anything but a novelty) [9] [10] so as to prevent the radius from concretely assuming a null value.

The evolution of the hypothesized Universe is evidently characterized by four consecutive phases: an accelerated expansion, a decelerated expansion, a decelerated contraction, an accelerated contraction. All the above-mentioned phases have the same duration.

By taking into account Equations (2.38), (2.39) and (2.41), we can immediately write the Hubble Parameter [11], commonly denoted by $H$, as follows:

$$
H=\frac{\dot{R}}{R}=\frac{c}{R_{m}} \frac{2 \sin \left(\frac{\alpha}{2}\right) \cos \left(\frac{\alpha}{2}\right)}{2 \sin ^{2}\left(\frac{\alpha}{2}\right)}=\frac{c}{R_{m}} \frac{1}{\tan \left(\frac{c t}{2 R_{m}}\right)} .
$$

\section{Introducing the $4^{\text {th }}$ Spatial Dimension}

\subsection{Mass-Energy "Equivalence": Alternative Derivation}

Let us consider a Material Point whose motion is defined by Equation (2.39) 
(in other terms, a simple harmonic oscillator consisting of a mass and an ideal spring).

Denoting with $m$ the mass of the above-mentioned point, taking into account Equation (2.36), the Elastic Constant, denoted by $k_{e}$, can be written as follows:

$$
k_{e}=m \omega^{2}=m\left(\frac{c}{R_{m}}\right)^{2}
$$

Consequently, the Total (Mechanical) Energy, with obvious meaning of the notation, acquires the following form:

$$
E_{R_{m} \text {-point }}=\frac{1}{2} k_{e} R_{m}^{2}=\frac{1}{2} m c^{2} .
$$

Now, by solely modifying the amplitude of the motion, denoted by $R_{m}^{\prime}$, keeping the values of mass and pulsation constant, we can generalize Equation (2.39) as follows:

$$
\left.\left.R^{\prime}=R^{\prime}\left(R_{m}^{\prime}, \alpha\right)=R_{m}^{\prime}(1-\cos \alpha), \quad R_{m}^{\prime} \in\right] 0, R_{m}\right] .
$$

From Equations (2.39) and (3.3) we have:

$$
\frac{R_{m}^{\prime}}{R_{m}}=\frac{R^{\prime}}{R}
$$

At any given time, the value of $R$ is obviously univocally determined by means of Equation (2.39), being $R_{m}$ constant. On the contrary, the value of $R^{\prime}$, provided by Equation (3.3), clearly depends on the amplitude of the motion $\left(R_{m}^{\prime}\right)$.

Taking into account Equations (3.1) and (3.4), the total energy of a material point, the motion of which is described by Equation (3.3), acquires the following form:

$$
E_{R_{m}^{\prime} \text {-point }}=\frac{1}{2} k_{e} R_{m}^{\prime 2}=\frac{1}{2}\left(\frac{R_{m}^{\prime}}{R_{m}}\right)^{2} m c^{2}=\frac{1}{2}\left(\frac{R^{\prime}}{R}\right)^{2} m c^{2} .
$$

The material point can now be replaced by a Material Homogeneous Segment (in other terms, it is as if we consider a spring, no longer ideal, with a length at rest equal to $R_{m}$ ). The length of the segment $(R)$ evolves in accordance to Equation (2.39).

If we denote now with $M$ the Mass of the Segment (the Linear Mass), the Linear Density can be banally defined as follows:

$$
\bar{M}=\frac{M}{R} \text {. }
$$

Consequently, denoting with $M^{\prime}$ the Mass of a Portion of Segment characterized, at any given time, by a length equal to $R^{\prime}$, we can write the following:

$$
M^{\prime}=\bar{M} R^{\prime}=\frac{R^{\prime}}{R} M
$$




$$
\bar{M}=\frac{M}{R}=\frac{M^{\prime}}{R^{\prime}} .
$$

Equation (3.8) clearly shows how the linear density does not vary along the segment. As a consequence, taking into account Equations (3.5) and (3.7), the Energy related to an Infinitesimal Material Segment can be written as follows:

$$
\mathrm{d} E_{R^{\prime}}=\frac{1}{2}\left(\frac{R^{\prime}}{R}\right)^{2} c^{2} \mathrm{~d} M^{\prime}=\frac{1}{2}\left(\frac{R^{\prime}}{R}\right)^{2} c^{2} \bar{M} \mathrm{~d} R^{\prime}=\frac{M c^{2}}{2 R^{3}} R^{\prime 2} \mathrm{~d} R^{\prime} .
$$

Taking now into account Equations (3.7) and (3.9), the final expression for the energy of a material segment, whose length, at any given time, is equal to $R^{\prime}$, acquires the underlying form:

$$
E_{R^{\prime}}=\int_{0}^{R^{\prime}} \mathrm{d} E^{\prime}=\frac{1}{6}\left(\frac{R^{\prime}}{R}\right)^{3} M c^{2}=\frac{1}{6}\left(\frac{R^{\prime}}{R}\right)^{2} M^{\prime} c^{2} .
$$

At this stage, it is necessary to introduce a Further Spatial Dimension [12].

The Universe we hypothesize is identifiable with a 4-Ball: the Radius, denoted by $R$, evolves in accordance to Equation (2.39). The corresponding boundary, that may represent the space we are allowed to perceive (at rest) [6] [12], is a three-dimensional surface (a Hyper Sphere) described by the following identity:

$$
x_{1}^{2}+x_{2}^{2}+x_{3}^{2}+x_{4}^{2}=R^{2} .
$$

The 4-Ball is banally described by the following inequality:

$$
x_{1}^{2}+x_{2}^{2}+x_{3}^{2}+x_{4}^{2} \leq R^{2} .
$$

Let us consider the point $P^{+}$defined as follows:

$$
P^{+}=(0,0,0, R) \text {. }
$$

Denoting with $P^{-}$the antipode of $P^{+}$(the point diametrically opposite), we have:

$$
P^{-}=(0,0,0,-R) .
$$

We must now consider the straight line segment bordered by the points $P^{+}$and $P^{-}$.

Figure 1 provides the representations of the above-mentioned segment, by looking into the scenarios that arise from Equation (3.12) if we set equal to zero, one at a time, all the four coordinates.

If we set $x_{4}=0$, we obtain nothing but a single point (as shown in Figure 1). Therefore, we have to examine the three-dimensional scenarios that arise from the underlying identity:

$$
x_{i}=0, i=1,2,3 .
$$

For example, we can set $x_{1}=0$ (obviously, the same line of reasoning can be followed by setting $x_{2}=0$ and $\left.x_{3}=0\right)$. As a consequence, from Equations (3.12), (3.13) and (3.14) we immediately obtain:

$$
x_{2}^{2}+x_{3}^{2}+x_{4}^{2} \leq R^{2},
$$




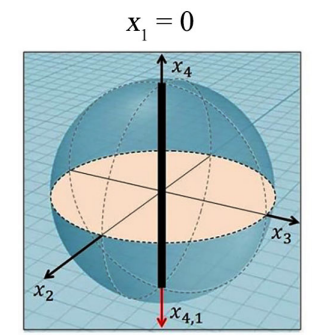

$x_{3}=0$
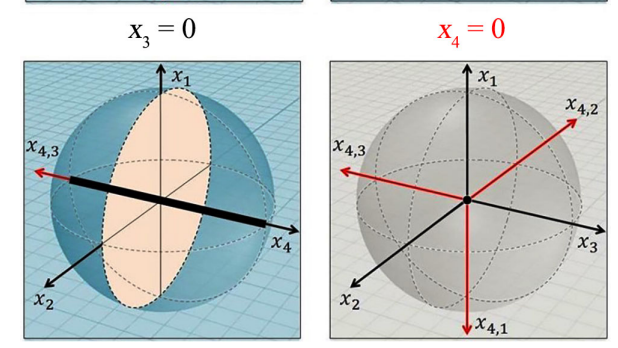

Figure 1. Representations of a material segment.

$$
\begin{aligned}
& P_{1}^{+}=(0,0, R), \\
& P_{1}^{-}=(0,0,-R) .
\end{aligned}
$$

Let us now consider the straight line segment bordered by the centre of the ball and the point defined by Equation (3.17). If the segment in question, the length of which evolves in accordance with (2.39), is provided with a mass equal to $M$, its energy can be immediately deduced from Equation (3.10) by setting $R^{\prime}=R$. Consequently, underlining how the same procedure can be obviously adopted for the point defined by Equation (3.18), we can write, with obvious meaning of the notation, as follows:

$$
\begin{gathered}
E_{R, 1}^{+}=E_{R, 1}^{-}=\frac{1}{6} M c^{2}, \\
E_{1}=E_{R, 1}=E_{R, 1}^{+}+E_{R, 1}^{-}=\frac{1}{3} M c^{2} .
\end{gathered}
$$

Generalizing, for the material segment, crossing the centre of the 4-Ball, characterized by a length equal to $2 R$ and a mass equal to $2 M$, we have:

$$
E_{i}=E_{R, i}=E_{R, i}^{+}+E_{R, i}^{-}=\frac{1}{3} M c^{2}, i=1,2,3 .
$$

Finally, by superposition, we can easily write the total amount of energy related to the material segment bordered by the points defined by Equations (3.13) and (3.14):

$$
E=\sum_{i=1}^{3} E_{i}=M c^{2}
$$

The points defined by Equations (3.13) and (3.14) are nothing but the interceptions between the material segment, the energy is provided by Equation (3.21), and the hyper surface described by Equation (3.11), which represents the Universe we are allowed to perceive (when we are at rest, as we will see later) [6] [12] [13]. 
As far as our perception of reality is concerned, each point and its antipode are to be actually considered as being the same entity, since they both belong to the same straight line segment. In other terms, we can state that, according to our model, the Universe is characterized by a concrete Global Central Symmetry. [6] [12] [13]

\subsection{The Conservation of Energy}

Let us consider one amongst the scenarios defined by Equation (3.15). For example, we can set, once again, $x_{1}=0$. Initially, the homogenous material segment, bordered by the points $P^{+}$and $P^{-}$defined in Equations (3.17) and (3.18), is characterized by a length equal to $2 R$ and a mass equal to $2 M$. Let us suppose that the segment starts rotating around the centre of the 3 -Ball defined by Equation (3.16).

If we impose the Conservation of Energy, the motion must necessarily entail modifications involving length and/or mass of the segment: otherwise the Kinetic Energy would be simply added to the energy defined by Equation (3.21), and the Total Energy could no longer be regarded as being constant. Obviously, the length of the segment in motion cannot increase: otherwise, the inequality in Equation (3.16) would be clearly violated (in other terms, the points $P^{+}$ and $P^{-}$would end up with being paradoxically placed beyond the boundary).

Now, in order to find an equation that may express the conservation of energy, we must impose some conditions.

Firstly, we impose that the Tangential Speed of the endpoints (of the segment), from now onwards denoted by $v$, cannot exceed the speed of light.

Secondly, we impose that the motion does not cause any linear density variations (this specific condition will be later legitimized): therefore, the value of the linear mass must keep on abiding by the simple rule established in Equation (3.7).

Ultimately, according to our model, the motion may produce, concurrently, a loss of linear mass and a (symmetric) reduction of the length of the segment.

If $2 R^{\prime}$ represents the total length of the segment, denoting with $I$ the Moment of Inertia, we can write the Kinetic Energy as follows:

$$
E_{k, 1}=\frac{1}{2} I\left(\frac{v}{R^{\prime}}\right)^{2} .
$$

If $2 M^{\prime}$ represents the (reduced) mass of the segment (in motion), we have:

$$
I=\frac{1}{12}\left(2 M^{\prime}\right)\left(2 R^{\prime}\right)^{2}=\frac{2}{3} M^{\prime} R^{\prime 2} .
$$

From the two previous Equations we immediately obtain:

$$
E_{k, 1}=\frac{1}{3} M^{\prime} v^{2}
$$

From Equation (3.10), taking into account the symmetry, we can state that 
the segment, since it is involved in the cyclic evolution described by Equation (3.3), is also provided with the following energy:

$$
E_{R^{\prime}, 1}=E_{R^{\prime}, 1}^{+}+E_{R^{\prime}, 1}^{-}=\frac{1}{3}\left(\frac{R^{\prime}}{R}\right)^{2} M^{\prime} c^{2}=E_{p, 1} .
$$

From Equations (3.25) and (3.26), taking into account the condition expressed in Equation (3.7), we obtain:

$$
E_{k, 1}+E_{p, 1}=\frac{1}{3} M^{\prime}\left[v^{2}+\left(\frac{R^{\prime}}{R}\right)^{2} c^{2}\right]=\frac{1}{3} M\left[v^{2}+\left(\frac{R^{\prime}}{R}\right)^{2} c^{2}\right] \frac{R^{\prime}}{R} .
$$

It is easy to verify how, since $V$ cannot exceed the speed of light, when $R^{\prime}$ approaches 0 (when the segment tends to completely lose its mass), $E_{k, 1}+E_{p, 1}$ tends to vanish. Therefore, in order to grant the conservation of energy, we need to introduce a further energetic term, denoted by $E_{w, 1}$. Obviously, when $R^{\prime}=R \quad$ (when $M^{\prime}=M$ ), $E_{w, 1}$ must vanish; on the contrary, when $R^{\prime}$ approaches 0 (when the segment tends to completely lose its mass), $E_{w, 1}$ must tend to the value in Equation (3.21). Moreover, since $E_{k, 1}+E_{p, 1}$ linearly depends on $M^{\prime}$, we impose a linear dependence also between $E_{w, 1}$ and $M^{\prime}$. Consequently, we have:

$$
E_{w, 1}=\frac{1}{3}\left(M-M^{\prime}\right) c^{2}
$$

Taking into account Equations (3.21), (3.25), (3.26) and (3.28), we can finally write the conservation of energy, for the considered scenario $\left(x_{1}=0\right)$, as follows:

$$
E_{1}=\frac{1}{3} M c^{2}=\frac{1}{3} M^{\prime} v^{2}+\frac{1}{3}\left(\frac{R^{\prime}}{R}\right)^{2} M^{\prime} c^{2}+\frac{1}{3}\left(M-M^{\prime}\right) c^{2}
$$

By multiplying by three all the members of the foregoing, taking into account Equation (3.22), we finally obtain the underlying relation:

$$
E=M c^{2}=M^{\prime} v^{2}+\left(\frac{R^{\prime}}{R}\right)^{2} M^{\prime} c^{2}+\left(M-M^{\prime}\right) c^{2}=E_{k}+E_{p}+E_{w} .
$$

$E_{k}$ represents the (real) kinetic energy, $E_{p}$ the Potential (Background) Energy (related to the cyclic evolution of the Universe), $E_{w}$ (a "non-material" aliquot, which may be related to the so-called “Quantum PotentiaP”) [14] [15], represents the energy needed to obtain the motion (to obtain the mass reduction).

From Equation (3.30) we immediately deduce the underlying noteworthy identity:

$$
M^{\prime} c^{2}=M^{\prime} v^{2}+\left(\frac{R^{\prime}}{R}\right)^{2} M^{\prime} c^{2}=E^{\prime}
$$

According to the definition of Lorentz Factor [16], we have:

$$
\gamma=\frac{1}{\sqrt{1-\left(\frac{v}{c}\right)^{2}}}
$$




$$
\left(\frac{v}{c}\right)^{2}=\beta^{2}=1-\frac{1}{\gamma^{2}} .
$$

From Equation (3.31), exploiting Equations (3.32) and (3.33), we easily obtain:

$$
R^{\prime}=R \sqrt{1-\left(\frac{v}{c}\right)^{2}}=R \sqrt{1-\beta^{2}}=\frac{R}{\gamma}
$$

We have just found the relation between the tangential speed (of the endpoints) and the Radial Extension (taking into account the symmetry) of the segment in motion.

From Equation (3.6), by virtue of the foregoing, we obtain:

$$
\frac{M}{R^{\prime}}=\frac{R}{R^{\prime}} \bar{M}=\frac{\bar{M}}{\sqrt{1-\left(\frac{v}{c}\right)^{2}}}=\gamma \bar{M} .
$$

Consequently, taking into account Equations (3.7), (3.8), (3.33), (3.34) and (3.35), the Specific Energies (the energies per unit of length) defined in Equation (3.30), can now be written, with obvious meaning of the notation, as follows:

$$
\begin{gathered}
\bar{E}=\frac{M c^{2}}{R^{\prime}}=\frac{\bar{M} c^{2}}{\sqrt{1-\left(\frac{v}{c}\right)^{2}}}=\gamma \bar{M} c^{2}, \\
\bar{E}_{k}=\frac{M^{\prime} v^{2}}{R^{\prime}}=\bar{M} \beta^{2} c^{2}=\left(1-\frac{1}{\gamma^{2}}\right) \bar{M} c^{2}, \\
\bar{E}_{p}=\left(\frac{R^{\prime}}{R}\right)^{2} \frac{M^{\prime} c^{2}}{R^{\prime}}=\frac{\bar{M} c^{2}}{\gamma^{2}}, \\
\bar{E}_{w}=\left(\frac{M}{M^{\prime}}-1\right) \frac{M^{\prime} c^{2}}{R^{\prime}}=\left(\frac{R}{R^{\prime}}-1\right) \frac{M^{\prime} c^{2}}{R^{\prime}}=(\gamma-1) \bar{M} c^{2} .
\end{gathered}
$$

Therefore, by dividing both members of Equation (3.30) by $R^{\prime}$, we obtain:

$$
\begin{gathered}
\bar{E}=\bar{E}_{k}+\bar{E}_{p}+\bar{E}_{w}, \\
\gamma \bar{M} c^{2}=\left(1-\frac{1}{\gamma^{2}}\right) \bar{M} c^{2}+\frac{\bar{M} c^{2}}{\gamma^{2}}+(\gamma-1) \bar{M} c^{2} .
\end{gathered}
$$

Denoting with $E_{0}$ the Energy at Rest $\left(R^{\prime}=R\right)$, provided by Equation (3.22), by virtue of the Equations (3.6), (3.36) and (3.39), we have:

$$
\begin{gathered}
\bar{E}_{0}=\frac{M c^{2}}{R}=\bar{M} c^{2}, \\
\bar{E}=\gamma \bar{M} c^{2}=\bar{E}_{0}+(\gamma-1) \bar{M} c^{2}=\bar{E}_{0}+\bar{E}_{w} .
\end{gathered}
$$

Now, by dividing both members of Equation (3.31) by $R^{\prime}$, taking into account Equations (3.8) and (3.34), we obtain:

$$
\bar{M} c^{2}=\bar{M} v^{2}+\frac{\bar{M} c^{2}}{\gamma^{2}} .
$$


By multiplying both members of the foregoing equation by $\gamma$, taking into account Equation (3.36), we have:

$$
\begin{gathered}
\gamma \bar{M} c^{2}=\gamma \bar{M} v^{2}+\frac{\bar{M} c^{2}}{\gamma} \\
\bar{E}=\frac{\bar{M} c^{2}}{\sqrt{1-\left(\frac{v}{c}\right)^{2}}}=\frac{\bar{M} v^{2}}{\sqrt{1-\left(\frac{v}{c}\right)^{2}}}+\sqrt{1-\left(\frac{v}{c}\right)^{2}} \bar{M} c^{2} .
\end{gathered}
$$

\section{3. "Relativistic Energy": Punctual Mass and Space Quanta}

In order to obtain the formal definition of the so-called Relativistic Energy, we have to impose a Space Quantization.

If $R$ is regarded as a Primary Measurable Quantity [17], denoting with $\Delta R_{\min }$ the (Radial) Quantum of Space [18] (the value of which will be estimated at a later time), and with $\mathcal{N}$ an integer (obviously, $\mathcal{N} \neq 0$ ), we can write:

$$
R=\mathcal{N} \Delta R_{\min }
$$

The Punctual (Three-Dimensional) Mass, denoted by $m$, can be defined as follows:

$$
m=\bar{M} \Delta R_{\min } .
$$

We can now finally legitimize the hypothesis of constancy of the linear density. From Equations (3.6) and (3.48), in fact, we can immediately deduce how the punctual mass is not influenced by the motion. In other terms, by virtue of the constancy of the linear density, $m$ can be considered as being constant (and the misleading concept of Relativistic Mass can be definitively rejected).

Now, taking into account Equation (3.40), for a Material Point we have:

$$
E_{m}=\bar{E} \Delta R_{\min }=\left(\bar{E}_{k}+\bar{E}_{p}+\bar{E}_{w}\right) \Delta R_{\min }=E_{k, m}+E_{p, m}+E_{w, m} .
$$

By multiplying both members of Equation (3.41) by $\Delta R_{\min }$, we have:

$$
E_{m}=\gamma m c^{2}=\left(1-\frac{1}{\gamma^{2}}\right) m c^{2}+\frac{m c^{2}}{\gamma^{2}}+(\gamma-1) m c^{2} .
$$

Now, by multiplying all the members of Equation (3.47) by $\Delta R_{\min }$, we finally obtain the well-known relation for the Relativistic Energy [3] [4]:

$$
E_{m}=\frac{m c^{2}}{\sqrt{1-\left(\frac{v}{c}\right)^{2}}}=\frac{m v^{2}}{\sqrt{1-\left(\frac{v}{c}\right)^{2}}}+\sqrt{1-\left(\frac{v}{c}\right)^{2}} m c^{2} .
$$

Denoting with $p_{m}$ the Momentum, with $\mathcal{L}$ the (Relativistic) Lagrangian, and with $\mathcal{H}$ the Hamiltonian, we have:

$$
p_{m}=\frac{m v}{\sqrt{1-\left(\frac{v}{c}\right)^{2}}},
$$




$$
\mathcal{L}=-\sqrt{1-\left(\frac{v}{c}\right)^{2}} m c^{2},
$$

Consequently, we can rewrite Equation (3.51) as follows:

$$
E_{m}=\mathcal{H}=p_{m} v-\mathcal{L} \text {. }
$$

\subsection{Towards a "New (Special) Relativity"}

According to the results up to now obtained, what we perceive as being a (material) point may actually be a straight line (material) segment crossing the center of the 4-Ball described by the inequality in Equation (3.12).

The endpoints represent all we are allowed to perceive of any segment. Coherently with the hypothesized central symmetry, moreover, the endpoints are to be considered as being a unique entity (in a certain sense, they can be regarded as "entangled").

The Uniform Linear Motion of a punctual mass may actually be a rotation (with a constant angular speed) of the corresponding material segment around the center of the 4-Ball. The rotation produce, concurrently, a loss of linear mass (although the value of the punctual mass is clearly preserved) and a (symmetric) reduction of the length: the new radial extension of the segment (half its length), denoted by $R^{\prime}$, depends on the value of the tangential speed acquired by its endpoints (the constant speed that characterize the apparent linear uniform motion, denoted by $v$ ).

The relation between $R^{\prime}$ and $v$ is expressed by Equation (3.34).

The Universe perceived by an observer involved in a linear uniform motion, characterized by a constant speed equal to $V$, is therefore described by the following equality:

$$
x_{1}^{2}+x_{2}^{2}+x_{3}^{2}+x_{4}^{2}=R^{\prime 2} \text {. }
$$

\section{The Lorentz Transformations}

The Lorentz Transformations can be considered, without any doubt, as the backbone of SR. Nonetheless, both the conventional derivation of the transformations and the meaning commonly assigned to them have been often savagely criticized, to the extent that, despite an alleged empirical evidence, the whole SR theory, in several occasions, has been brought into question.

Firstly, it is worth underlining how, as Lorentz himself was forced to admit at a later time [19], the transformations had been already conceived, several years before the publication of the famous paper [16], by someone else [20]. Secondly, the work of Lorentz was anything but concretely linked to relativistic issues, at least in the Einsteinian sense of the term [3] [4]. Very simply, Lorentz's aim fundamentally lay in finding some transformations able to formally make the well-known Maxwell Equations [21] (Maxwell, 1873) invariant. On this subject, moreover, it can be even proved how the Lorentz transformations are not the only ones able to preserve the formal validity of the Maxwell equations [22].

The so-called Direct Transformations [16] are usually written, with obvious 
meaning of symbols and signs, as follows:

$$
\begin{aligned}
& x=\frac{x^{\prime}+v t^{\prime}}{\sqrt{1-\left(\frac{v}{c}\right)^{2}}} \\
& t=\frac{t^{\prime}+\frac{v x^{\prime}}{c^{2}}}{\sqrt{1-\left(\frac{v}{c}\right)^{2}}} .
\end{aligned}
$$

The so-called Inverse Transformation [16] are usually written in the following form:

$$
\begin{aligned}
& x^{\prime}=\frac{x-v t}{\sqrt{1-\left(\frac{v}{c}\right)^{2}}}, \\
& t^{\prime}=\frac{t-\frac{v x}{c^{2}}}{\sqrt{1-\left(\frac{v}{c}\right)^{2}}} .
\end{aligned}
$$

It is commonly said that, when the speed assumed by the mobile frame of reference is far less than that of light, the Lorentz Transformations tend to the Galilean ones. In other terms, according to the previous assertion, Galilean Relativity should be interpreted as a particular case of the Einsteinian one. This is an erroneous conviction [23]. In fact, referring to the ratio that appears in the numerator of Equations (4.2) and (4.4), it is easy to understand how no limitation turns out to be imposed, respectively, on the variables $x$ and $x^{\prime}$. Therefore, since the above mentioned variables should be able to evidently assume arbitrarily large values, the ratio we have taken into consideration could even not tend to zero, so making de facto impossible a real identification of the Lorentz transformations with the Galilean ones [24]. As we are about to see, however, this misleading problem can be easily overcome by means of an alternative derivation of the transformations, carried out by imposing the absoluteness of time.

\subsection{The Lorentz Transformations: Alternative Derivation}

In order to deduce the direct transformations, we will consider the scenario in Figure 2.

The deduction will be carried out net of the symmetry.

We denote with $O$ the origin of the Frame of Reference at Rest, and with $O^{\prime}$ the origin of the Frame of Reference in Motion. At the beginning, obviously, $O$ and $O^{\prime}$ coincide. We have to hypothesize that when $O^{\prime}$ starts moving, with a constant speed equal to $V$, a signal is simultaneously sent from a source, that both the observer at rest and the one in motion will perceive as being punctual. The initial Angular Distance between the origins and the source is denoted by $\chi$. 


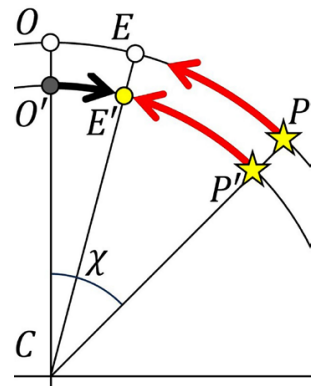

Figure 2. Direct transformations.

The signal is actually sent from each of the points that belong to the straight line segment bordered by the center of curvature, denoted by $C$, and $P$. The latter represents the source as perceived by an observer at rest. The radial extension of any point at rest is evidently equal to the radius of the 4-Ball, denoted by $R$.

As soon as $O^{\prime}$ starts moving, its radial extension, denoted by $R^{\prime}$, assumes the value provided by Equation (3.34). If we denote with $l_{O P}$ the arc bordered by $O$ and $P$, representing the distance at rest from the source, and with $l_{O^{\prime} P^{\prime}}$ the arc bordered by $O^{\prime}$ and $P^{\prime}$, which represents the distance between $O^{\prime}$ and the source as soon as the motion occurs, taking into account Equation (3.34), we can write the following:

$$
\begin{gathered}
\overline{C O}=\overline{C P}=R, \\
\overline{C O^{\prime}}=\overline{C P^{\prime}}=R^{\prime}, \\
l_{O P}=R \chi, \\
l_{O^{\prime} P^{\prime}}=R^{\prime} \chi, \\
\frac{l_{O P}}{l_{O^{\prime} P^{\prime}}}=\frac{R}{R^{\prime}}=\frac{1}{\sqrt{1-\left(\frac{v}{c}\right)^{2}}}, \\
l_{O P}=\frac{l_{O^{\prime} P^{\prime}}}{\sqrt{1-\left(\frac{v}{c}\right)^{2}}} .
\end{gathered}
$$

The coordinate of the light source as measured by the observer at rest, up until now denoted by $l_{O P}$, can be replaced by $x$. After a certain time, denoted by $t^{\prime}$, the observer in motion intercepts the signal. Let's denote with $E^{\prime}$ the rendezvous point. Obviously, the time elapsed is equal to the time taken by light to cover the distance $l_{E^{\prime} P^{\prime}}$. The above mentioned distance coincides with the coordinate of the light source, denoted by $x^{\prime}$, as measured by the observer in motion as soon as the signal is received. We have:

$$
\begin{gathered}
l_{O P}=x, \\
l_{E^{\prime} P^{\prime}}=x^{\prime}, \\
t^{\prime}=\frac{l_{E^{\prime} P^{\prime}}}{c}=\frac{x^{\prime}}{c},
\end{gathered}
$$




$$
\begin{gathered}
l_{O^{\prime} E^{\prime}}=v t^{\prime}, \\
l_{O^{\prime} P^{\prime}}=l_{E^{\prime} P^{\prime}}+l_{O^{\prime} E^{\prime}}=x^{\prime}+v t^{\prime} .
\end{gathered}
$$

From Equations (4.10) and (4.15) we can immediately deduce (4.1), which represents the First Direct Lorentz Transformation.

If we divide the first and second member of Equation (4.1) by $c$, we obtain:

$$
\frac{x}{c}=\frac{\frac{x^{\prime}}{c}+\frac{v t^{\prime}}{c}}{\sqrt{1-\left(\frac{v}{c}\right)^{2}}} .
$$

The first member of the previous equation, that can be denoted by $t$, represents the time elapsed between the light signal emission and the moment in which the observer at rest succeeds in seeing it. From Equations (4.13) and (4.16) we can immediately obtain Equations (4.2), that represents the Second Direct Lorentz

\section{Transformation.}

In order to deduce the inverse transformations, we will consider the scenario in Figure 3.

This time, referring to the bi-dimensional representation provided by Figure 3 , we have to suppose that the occurs counterclockwise (once again, with a constant speed equal to $v$ ). Obviously, the Equations from (4.5) to (4.10) are still valid. We can serenely exploit the line of reasoning previously followed in deriving the direct transformations, being careful to switch the superscripts: from the point of view of the observer in motion, in fact, the one at rest, placed in $O$, seems to approach the light source (moving with a constant speed equal to $v$ ).

Therefore, we can now write the following:

$$
\begin{gathered}
l_{O P}=x^{\prime}, \\
l_{E^{\prime} P^{\prime}}=x, \\
t=\frac{l_{E^{\prime} P^{\prime}}}{c}=\frac{x}{c}, \\
l_{E^{\prime} O^{\prime}}=v t, \\
l_{O^{\prime} P^{\prime}}=l_{E^{\prime} P^{\prime}}-l_{E^{\prime} O^{\prime}}=x-v t .
\end{gathered}
$$

From Equations (4.10) and (4.21) we can immediately deduce Equation (4.3), which represents the First Inverse Lorentz Transformation.

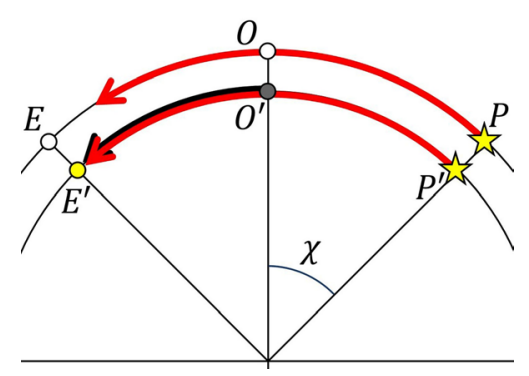

Figure 3. Inverse transformations. 
If we divide the first and second member of Equation (4.3) by $c$, we obtain:

$$
\frac{x^{\prime}}{c}=\frac{\frac{x}{c}-\frac{v t}{c}}{\sqrt{1-\left(\frac{v}{c}\right)^{2}}} .
$$

The first member of the previous equation, that can be denoted by $t^{\prime}$, represents the time elapsed between the light signal emission and the moment in which the observer placed in $O$, actually at rest but considered as being in relative motion towards the source, succeeds in seeing it. From Equations (4.21) and (4.22) we can immediately obtain Equation (4.4), which represents the Second Direct Lorentz Transformation.

It is fundamental to underline that, if we take into account the symmetry, both the direct transformations and the inverse ones can be simultaneously applied to whatever point in motion with a constant speed equal to v. referring to Figure 4 (which represents just a modified version of the figure used to deduce the direct transformations), in fact, we can easily notice how, due to the symmetry, the light signals start not only from $P^{+}$and $P^{\prime+}$, but also from $P^{-}$and $P^{\prime-}$, moving both clockwise and counterclockwise.

Very simply, the observer in motion travels towards the signal that propagates counterclockwise, so making possible the adoption of the direct transformations; simultaneously, the same observer moves away from the signal that propagates clockwise, so making possible the adoption of the inverse transformations.

\subsection{Some Noteworthy Consequences}

Firstly, referring to both the previously described scenarios, we can state that if the motion were suddenly stopped in $E^{\prime}$, the traveler would be instantaneously dragged into $E$, and the signal, after a certain period of time, would be seen once again: in other terms, the observer would be involved in some sort of déjà-vu.

Secondly, we can state that the distance between the traveler and the light source undergoes a reduction as soon as the motion takes place: the higher the value of the speed, the higher the entity of the reduction. For example, referring to the first of the two cases previously examined, we can state that the traveler is able to cover the distance $l_{O^{\prime} P^{\prime}}$ by taking a time, denoted by $t_{m o b}$, provided by the following relation:

$$
t_{m o b}=\frac{l_{O^{\prime} P^{\prime}}}{v} .
$$

However, once the traveler reaches the light source, the observer at rest believes that the covered distance may be equal to $l_{O P}$. As a consequence, from the point of view of the observer at rest, the Apparent Speed of the traveler, denoted by $v_{a p p}$, is provided by the following relation:

$$
v_{a p p}=\frac{l_{O P}}{t_{m o b}}=\frac{l_{O P}}{l_{O^{\prime} P^{\prime}}} v .
$$




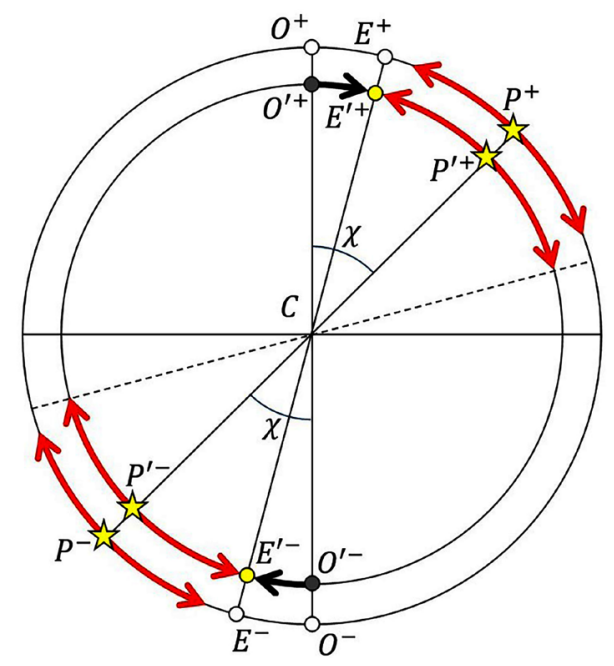

Figure 4. Symmetry.

From Equation (4.24), by taking into account Equation (4.9), we can immediately write the following:

$$
v_{a p p}=\frac{v}{\sqrt{1-\left(\frac{v}{c}\right)^{2}}} .
$$

Coherently with the domain of the relativistic factor, the Real Speed, that keeps on being denoted by $v$, can never equate that of light. On the contrary, the virtual speed, that we have denoted with $v_{a p p}$, tends to infinity when the real speed tends to that of light. This result is qualitatively described by Figure 5 , in which the $\mathrm{x}$-coordinate represents the ratio between the real speed and that of light $(\beta)$, and the $y$-coordinate represents the correspondent value of the virtual speed, in number of times that of light.

As a consequence, an observer at rest will measure, in any given case, a speed greater than the real one. Obviously, from Equation (4.25) it is possible to easily deduce the relation that expresses the virtual speed, the one measured by the observer at rest, as a function of the real one:

$$
v=\frac{v_{a p p}}{\sqrt{1+\left(\frac{v_{a p p}}{c}\right)^{2}}} .
$$

Let's now choose a "destination". Generalizing Equation (4.10), if we denote with $I$ the distance at rest from the point that we have to reach, and with $l_{m o b}$ the corresponding Reduced Distance (the distance that a traveler, who starts moving with a speed equal to $v$, should actually cover in order to reach the destination), we have:

$$
l_{m o b}=l \sqrt{1-\left(\frac{v}{c}\right)^{2}} .
$$




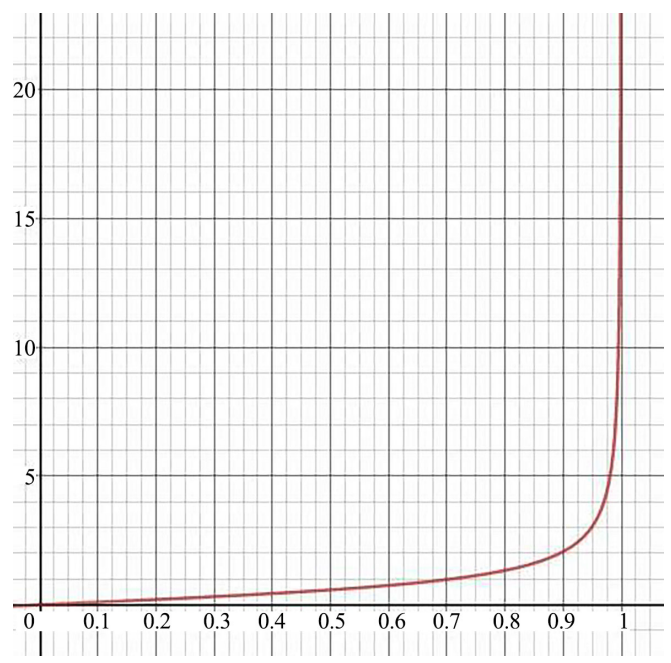

Figure 5. Apparent speed as a function of real speed.

This result is qualitatively described by Figure 6 in which the $\mathrm{x}$-coordinate represents, once again, the ratio between the real speed and that of light $(\beta)$, and the $y$-coordinate represents the correspondent value of the ratio between the reduced distance and the one measured at rest.

\subsection{The Alleged Increase of the Lifetime of Muons (Short Account)}

Amongst the so-called "proofs of Special Relativity", the alleged increase of the lifetime of muons stands out. In the light of the results up to now obtained, the phenomenon may be easily explained avoiding time dilations. Muons evidently succeed in covering a distance clearly not compatible with their mean lifetime: this is irrefutable. On the one hand, we could admit that time, for muons, starts slowing down due to the high value of their speed; on the other hand, and for the same reason, we may also imagine that, for muons, both the radial extension and the distances undergo a reduction (the phenomenon, according to our theory, is no longer restricted to the direction of the motion). In the latter case, the speed perceived by an observer at rest is greater than what it really is, and time, clearly, does not undergo any dilation whatsoever.

\section{Again on the Friedmann-Lemaître Equations}

The broad compatibility between our model of Universe and GR has been already addressed. On this subject, it is forth specifying how the above-mentioned compatibility is to be meant as being exclusively referred to each of the three-dimensional scenarios that arise from Equation (3.15): GR, in fact, is clearly a $3 D+1$ theory (at least in its original formulation) [3], while the Universe herein hypothesized is evidently based on a $4 D+1$ model. As a consequence, a direct comparison between our theory and GR turns out to be de facto impossible. 


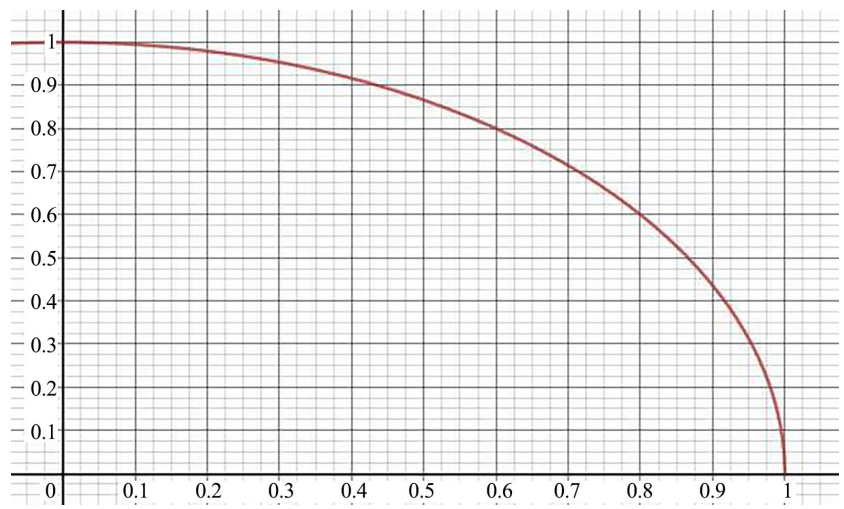

Figure 6. Reduced distance as a function of real speed.

In this section we carry out an alternative deduction (without resorting to GR) of the Friedmann-Lemaitre Equations [2]. It is nothing but an "inverse procedure", the finality of which exclusively lies in clarifying some positions, such as the one in Equation (2.35), we have previously exploited in building our model.

\subsection{The Friedmann-Lemaître Equations: Alternative Derivation}

By virtue of the global (central) symmetry [6] [12] [13] so far hypothesized, denoting with $M$ half the mass of the Universe, we can define the density as follows:

$$
\rho=\frac{M}{\frac{2}{3} \pi R^{3}} .
$$

Coherently with Equation (2.35), taking into account Equation (2.39), if $M_{m}$ represents the mass when $R=R_{m}$, from the prior equation we obtain:

$$
\rho_{m}=\rho\left(R_{m}\right)=\frac{M_{m}}{\frac{2}{3} \pi R_{m}^{3}}=\frac{3 \frac{c^{2}}{R_{m}^{2}}}{4 \pi \frac{c^{2} R_{m}}{2 M_{m}}} .
$$

Coherently with Equation (2.37), we can now carry out the following position:

$$
G=\frac{R_{m} c^{2}}{2 M_{m}} .
$$

From the previous position, taking into account Equation (5.2), we obtain:

$$
\rho_{m}=\frac{3 \frac{c^{2}}{R_{m}^{2}}}{4 \pi G} .
$$

We can now define the cosmological constant as follows:

$$
\Lambda=-\frac{3}{R_{m}^{2}} \text {. }
$$


Taking into account the foregoing, from Equation (5.4) we recover Equation (2.30).

Now, denoting with $p$ the pressure, and with $\sigma$ the Surface Tension (which must be clearly considered as being constant), we can write the well-known Young-Laplace Equation [25] as follows:

$$
p=\frac{2 \sigma}{R} .
$$

From the foregoing, by virtue of Equation (2.10), we can write:

$$
\frac{\mathrm{d} \sigma}{\mathrm{d} t}=\frac{1}{2} \frac{\mathrm{d}}{\mathrm{d} t}(p R)=(v-1) c^{2} \frac{\mathrm{d}}{\mathrm{d} t}(\rho R)=0 .
$$

From the previous, we deduce Equation (2.32) or, equivalently, the following:

$$
\rho=\frac{R_{m}}{R} \rho_{m}
$$

According to our hypotheses, moreover, we have:

$$
\begin{gathered}
\frac{\mathrm{d}}{\mathrm{d} t}\left(p V^{v}\right)=(v-1) \frac{\mathrm{d}}{\mathrm{d} t}\left(\rho V^{v}\right)=0, \\
\rho V^{v}=\rho_{m} V_{m}^{v} \\
\rho R^{3 v}=\rho_{m} R_{m}^{3 v} .
\end{gathered}
$$

By carrying out a banal comparison between Equations (5.8) and (5.11), we can finally set $v=1 / 3$. Consequently, from Equation (2.10) we immediately obtain:

$$
p=-\frac{2}{3} \rho c^{2}
$$

From Equations (5.2) and (5.8) we immediately obtain:

$$
\begin{gathered}
\rho=\frac{R_{m}}{R} \rho_{m}=\frac{3}{4 \pi G} \frac{c^{2}}{R R_{m}}, \\
\frac{c^{2}}{R R_{m}}=\frac{4 \pi G}{3} \rho .
\end{gathered}
$$

From Equations (2.40 and (2.41) we have:

$$
\dot{R}^{2}=c^{2}\left(1-\cos ^{2} \alpha\right)=2 c^{2} \frac{R}{R_{m}}-c^{2} \frac{R^{2}}{R_{m}^{2}} .
$$

If $R \neq 0$, from the previous, by virtue of Equations (5.5) and (5.14), we have:

$$
\begin{gathered}
\left(\frac{\dot{R}}{R}\right)^{2}+\frac{c^{2}}{R_{m}^{2}}=\left(\frac{\dot{R}}{R}\right)^{2}-\frac{\Lambda c^{2}}{3}=2 \frac{c^{2}}{R R_{m}}=\frac{8 \pi G}{3} \rho, \\
\left(\frac{\mathrm{d} R}{\mathrm{~d} t}\right)^{2}=\frac{1}{3}\left(8 \pi G \rho+\Lambda c^{2}\right) R^{2} .
\end{gathered}
$$


Evidently, the previous equation is nothing but Equation (2.1) with $k=0$. Now, Equation (5.15) can be easily rearranged as follows:

$$
\dot{R}^{2}=2 R \frac{c^{2}}{R_{m}}\left(1-\frac{R}{R_{m}}\right)+c^{2} \frac{R^{2}}{R_{m}^{2}} .
$$

From the foregoing, by virtue of Equations (2.42) and (5.5), if $R \neq 0$ we have:

$$
\begin{gathered}
\dot{R}^{2}=2 R \ddot{R}+c^{2} \frac{R^{2}}{R_{m}^{2}}=2 R \ddot{R}-\frac{\Lambda c^{2}}{3} R^{2}, \\
\left(\frac{\dot{R}}{R}\right)^{2}=2 \frac{\ddot{R}}{R}-\frac{\Lambda c^{2}}{3} .
\end{gathered}
$$

From Equations (5.12) and (5.16) we obtain:

$$
\begin{gathered}
\left(\frac{\dot{R}}{R}\right)^{2}-\frac{\Lambda c^{2}}{3}=-\frac{4 \pi G}{c^{2}} p \\
2\left(\frac{\dot{R}}{R}\right)^{2}-\frac{2}{3} \Lambda c^{2}=\left(\frac{\dot{R}}{R}\right)^{2}+\left(\frac{\dot{R}}{R}\right)^{2}-\frac{2}{3} \Lambda c^{2}=-\frac{8 \pi G}{c^{2}} p .
\end{gathered}
$$

From the previous, by taking into account Equation (5.20), we finally obtain the usual writing of the Second Friedmann-Lemaittre Equation [2] [3]:

$$
2 \frac{\ddot{R}}{R}+\left(\frac{\dot{R}}{R}\right)^{2}-\Lambda c^{2}=-\frac{8 \pi G}{c^{2}} p .
$$

\subsection{The Metric Expansion/Contraction as an Apparent Phenomenon}

Actually, we consider the variations of cosmological distances as being an apparent phenomenon: in other terms, we postulate that the amount of space between whatever couple of points remains the same with the passing of time (on this subject, it could be worth bearing in mind how Hubble himself started bringing into question the relation between the Redshift and the Recessional Velocity of Astronomical Objects) [26].

More precisely, we hypothesize that the so-called Cosmological Redshift may banally related to the conservation of energy.

As is well known, the energy of a Quantum of Light can be expressed as the product between the value of its frequency and the Plank Constant.

On the one hand, as an alternative to the conventional interpretation of the cosmological redshift, we could accept that, in travelling through the interstellar vacuum, light may somehow "get tired", so losing part of its energy [27] [28] [29].

On the other hand, we may simply imagine that the Plank Constant could vary over time [30] [31] [32]: consequently, just in order to preserve its energy, a photon could be forced into modifying its frequency. 
In the latter case, evidently, all the cosmological equations can be rewritten as a function a Plank Parameter.

On this subject, it can be proved that, according to the Generalized Uncertainty Principle, $\Delta R_{\min }$ in Equation (3.47) can be expressed as follows [17]:

$$
\Delta R_{\min }=2 \sqrt{\alpha^{\prime}} \ell_{P} .
$$

In the previous, $\ell_{P}$ represents the so-called Planck Length and $\alpha^{\prime}$ a constant.

There are several methods to estimate the value of $\alpha^{\prime}$ [33] [34] [35] [36]: in any case, however, we obtain $\alpha^{\prime} \cong 1$. Consequently, from Equations (3.47) and (5.24), making explicit the expression of $\ell_{P}$ and setting $\alpha^{\prime}=1$, we obtain:

$$
R=\mathcal{N} \Delta R_{\min }=2 \mathcal{N} \sqrt{\frac{\hbar G}{c^{3}}} .
$$

From the previous, taking into account Equation (2.43), we can easily deduce:

$$
\left(\frac{\dot{R}}{R}\right)^{2}=H^{2}=\frac{1}{4}\left(\frac{\dot{h}}{h}\right)^{2} .
$$

Therefore, Equation (5.17) can be rewritten, by resorting to the foregoing, as follows:

$$
\left(\frac{\mathrm{d} h}{\mathrm{~d} t}\right)^{2}=\frac{4}{3}\left(8 \pi G \rho+\Lambda c^{2}\right) h^{2} .
$$

Beyond doubt, the possible variability of the Planck constant could still sound like a shocking hypothesis: nonetheless, it is worth bearing in mind how several physical quantities, initially considered as being constant, have been later classified as variables. The Hubble constant faced exactly this fate, and quite soon it was downgraded, so to say, to the rank of parameter (the value of which is still under investigation) [37] [38] [39].

\section{Gravitation: A Simple Model}

In the light of what specified in the sub-paragraph 5.2, $R_{m}$ and $M_{m}$ can be conventionally considered, respectively, as the real values of radius and mass.

Replacing, for convenience, $R_{m}$ with $R_{s}$, and $M_{m}$ with $M_{t o t}$ (half the real mass of the Universe, coherently with the symmetry), we now can rewrite Equation (2.37):

$$
R_{s}=\frac{2 G M_{t o t}}{c^{2}} .
$$

Obviously, Equations (3.11) and (3.12) assume the following form:

$$
\begin{aligned}
& x_{1}^{2}+x_{2}^{2}+x_{3}^{2}+x_{4}^{2}=R_{s}^{2}, \\
& x_{1}^{2}+x_{2}^{2}+x_{3}^{2}+x_{4}^{2} \leq R_{s}^{2} .
\end{aligned}
$$

Let us denote with $C$, as usual, the centre of the 4-Ball (with which we identify the Universe in its entirety), with $O$ and $P$ two points, the first of 
which taken as origin, placed on the hyper-surface defined in Equation (6.2), and with $O^{\prime}$ the centre of the so-called Measured Circumference, to which $P$ belongs. Both $O$ and $O^{\prime}$ are considered as lying on $x_{4}$. The angular distance between $O$ and $P$, as perceived by an ideal observer placed in $C$, is denoted by $\chi$.

The arc bordered by $O$ and $P$, denoted by $R_{p}$, represents the so-called Proper Radius (the measured distance between the above-mentioned points). We have:

$$
R_{p}(\chi)=R_{s} \chi
$$

The straight-line segment bordered by $O^{\prime}$ and $P$, denoted by $R_{c}$ or $X$, represents the so-called Predicted (or Forecast) Radius (the ratio between the perimeter of the measured circumference and $2 \pi$ ). We have:

$$
R_{c}(\chi)=X(\chi)=R_{s} \sin \chi .
$$

From the previous we immediately deduce:

$$
\chi=\arcsin \left(\frac{X}{R_{s}}\right)
$$

Consequently, taking into account Equation (6.4), we have:

$$
\mathrm{d} R_{p}=R_{s} \mathrm{~d} \chi=\frac{\mathrm{d} X}{\sqrt{1-\left(\frac{X}{R_{s}}\right)^{2}}} .
$$

The scenario is qualitative depicted in Figure 7.

At this point, for the hyper-surface defined in Equation (6.2), the so-called Friedmann-Lemaittre-Robertson-Walker Metric (with $k=1$ ) [2] [3] can be written:

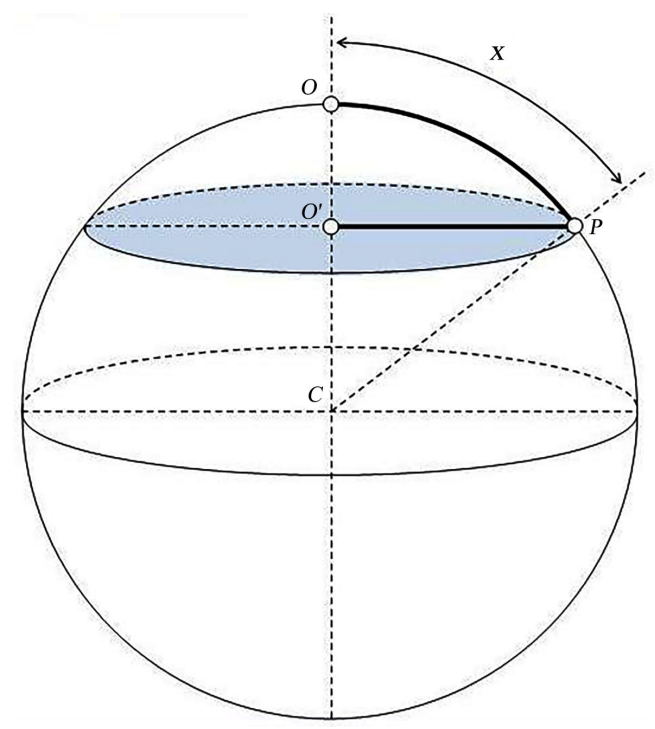

Figure 7. Proper radius, predicted radius and measured circumference. 


$$
\mathrm{d} s^{2}=c^{2} \mathrm{~d} t^{2}-\frac{\mathrm{d} X^{2}}{1-\left(\frac{X}{R_{s}}\right)^{2}}-X^{2}\left(\mathrm{~d} \theta^{2}+\sin ^{2} \theta \mathrm{d} \varphi^{2}\right) .
$$

Let us denote with $S_{2}$ the 2-Sphere characterized by a radius of curvature equal to $R_{c}$. In order to simplify the notation, from now onwards we shall denote with the same symbol both the geometrical object and the corresponding surface area or volume. Denoting, as usual, with $\theta$ the Inclination and with $\varphi$ the Azimuth, taking into account Equation (6.5), we have:

$$
S_{2}(\chi)=X^{2} \int_{\varphi=0}^{2 \pi} \int_{\theta=0}^{\pi} \sin \theta \mathrm{d} \theta \mathrm{d} \varphi=X^{2} \int_{0}^{4 \pi} \mathrm{d} \Omega=4 \pi X^{2}=4 \pi R_{s}^{2} \sin ^{2} \chi .
$$

The above-mentioned surface is simultaneously border of a 3-Ball, denoted by $V_{3}$, and of a Hyper-Spherical Cap, denoted by $S_{3} . V_{3}$ represents the Predicted (or Forecast) Volume, $S_{3}$ the Proper Volume. From Equations (6.4), (6.5) and (6.9), we have:

$$
\begin{gathered}
V_{3}(\chi)=\int_{0}^{R_{c}} S_{2}(\chi) \mathrm{d} R_{c}=4 \pi \int_{0}^{X} X^{2} \mathrm{~d} X=\frac{4}{3} \pi X^{3}=\frac{4}{3} \pi R_{s}^{2} \sin ^{3} \chi, \\
S_{3}(\chi)=\int_{0}^{R_{c}} S_{2}(\chi) \mathrm{d} R_{p}=4 \pi R_{s}^{3} \int_{0}^{\chi} \sin ^{2} \chi \mathrm{d} \chi=2 \pi R_{s}^{3}(\chi-\sin \chi \cos \chi) .
\end{gathered}
$$

We can generalize the foregoing as follows:

$$
S_{3}\left(R^{\prime}, \chi\right)=2 \pi R^{\prime 3}(\chi-\sin \chi \cos \chi), R^{\prime} \in\left[0, R_{s}\right] .
$$

The Hyper-Spherical Cap $S_{3}$, defined in Equation (6.11), is clearly associated to a Hyper-Spherical Sector, denoted by $V_{4}$. We have:

$$
\begin{aligned}
V_{4}(\chi) & =\int_{0}^{R_{s}} S_{3}\left(R^{\prime}, \chi\right) \mathrm{d} R^{\prime}=2 \pi(\chi-\sin \chi \cos \chi) \int_{0}^{R_{s}} R^{\prime 3} \mathrm{~d} R^{\prime} \\
& =\frac{1}{2} \pi R_{s}^{4}(\chi-\sin \chi \cos \chi) .
\end{aligned}
$$

\subsection{Gravitational "Singularities": Four-Dimensional Scenario}

As previously stated, the (curved) space we are allowed to perceive can be approximately identified with a Hyper-Sphere, the radius of which depends on our state of motion: at rest, according to Equation (3.34), this radius equates $R_{s}$.

In our simple model the total amount of mass is constant: in other terms, mass can only be redistributed. Let us consider a generic point $Q$, belonging to the surface of the 4-Ball, and let us denote with $\chi_{\max }$ the angular distance between this point and the origin $O$. In order to create a Gravitational "Singularity" in correspondence of the origin, we have to ideally concentrate in $O$, from the point of view of an observer at rest (who is exclusively allowed to perceive a three-dimensional curved universe), all the mass enclosed in the 2 -Sphere defined by Equation (6.9) (with $\chi=\chi_{\max }$ ). This surface represents the border of the Hyper-Spherical Cap defined in Equation (6.11) (with $\left.\chi=\chi_{\max }\right)$ which, in turn, is associated to the Hyper-Spherical Sector defined in Equation (6.13) (with $\chi=\chi_{\max }$ ). 
According to our theory, in enacting the ideal procedure previously expounded, we actually hypothesize that all the mass of the Hyper-Spherical Sector earlier defined may be concentrated (and evenly spread) along the material segment bordered by $C$ and $O$. The procedure entails a linear mass (energy) density increment, no longer compatible with the previous radial extension: consequently, both the segment and the corresponding space undergo a radial contraction (the segment shortens together with space) and the surrounding spatial "lattice", the integrity of which must be in any case preserved, results deformed. We want to determine the new radial extension of the segment (that represents the singularity) and the shape of the deformed spatial lattice.

It is worth specifying how, abiding to the global central symmetry previously introduced, the procedure just described is symmetric with respect to the centre of the 4-Ball: consequently, we should have actually considered two opposite Hyper-Spherical Sectors, characterized by the same amplitude, and a single material segment, crossing the centre $C$, bordered by $O$ and its antipodal point.

\subsection{Gravitational "Singularities": Three-Dimensional Scenario}

From Equation (6.3), by setting equal to zero, one at a time, $x_{1}, x_{2}$ and $x_{3}$, we obtain the following three-dimensional scenarios:

$$
\begin{aligned}
& x_{4,1}^{2}+x_{2}^{2}+x_{3}^{2} \leq R_{s}^{2}, \\
& x_{1}^{2}+x_{4,2}^{2}+x_{3}^{2} \leq R_{s}^{2}, \\
& x_{1}^{2}+x_{2}^{2}+x_{4,3}^{2} \leq R_{s}^{2} .
\end{aligned}
$$

Evidently, if we take into consideration one among the static scenarios we have just obtained, the procedure previously discussed (the creation of the singularity) is equivalent to concentrating along a segment the mass of a spherical sector.

Taking into account Equation (6.5), let us denote with $S_{2-1}$, the Circumference defined by the following relation:

$$
S_{2-1}(\chi)=2 \pi X .
$$

In the three-dimensional scenario we have been considering, $S_{2-1}$ "plays the role" of $S_{2}$, defined in Equation (6.9).

The circumference defined in Equation (6.17) is simultaneously border of a Disc, denoted by $V_{3-1}$, and of a Spherical Cap, denoted by $S_{3-1}$. In the threedimensional scenario we have been considering, the first "plays the role" of the Predicted (or Forecast) Volume $V_{3}$, defined in Equation (6.10), while the second "plays the role" of the Proper Volume $S_{3}$, defined in Equation (6.11). We have:

$$
V_{3-1}(\chi)=\int_{0}^{R_{c}} S_{2-1}(\chi) \mathrm{d} R_{c}=2 \pi \int_{0}^{X} X \mathrm{~d} X=\pi X^{2}=\pi R_{s}^{2} \sin ^{2} \chi,
$$




$$
S_{3-1}(\chi)=\int_{0}^{R_{p}} S_{2-1}(\chi) \mathrm{d} R_{p}=2 \pi \int_{0}^{\chi} \sin \chi \mathrm{d} \chi=2 \pi R_{s}^{2}(1-\cos \chi)
$$

We can generalize the foregoing as follows:

$$
S_{3-1}\left(R^{\prime}, \chi\right)=2 \pi R^{\prime 2}(1-\cos \chi), R^{\prime} \in\left[0, R_{s}\right]
$$

The Spherical Cap $S_{3-1}$, defined in Equation (6.19), is clearly associated to a Spherical Sector, denoted by $V_{4-1}$. We have:

$$
\begin{aligned}
V_{4-1}(\chi) & =\int_{0}^{R_{s}} S_{3-1}\left(R^{\prime}, \chi\right) \mathrm{d} R^{\prime}=2 \pi(1-\cos \chi) \int_{0}^{R_{s}} R^{\prime 2} \mathrm{~d} R^{\prime} \\
& =\frac{2}{3} \pi R_{s}^{3}(1-\cos \chi) .
\end{aligned}
$$

In the three dimensional scenario we have been considering, $V_{4-1}$ "plays the role" of $V_{4}$, defined in Equation (6.13).

As previously highlighted, the new radial extension of the segment (that represents the singularity) is still unknown, as well as the shape of the deformed spatial lattice.

Let us denote with $r$ the Radial Coordinate of a generic point of the warped surface.

According the well-known Shell Theorem, a spherically symmetric body affects the external particles as if its entire mass were concentrated at the centre. Therefore, coming back for a while to the four-dimensional scenario and limiting our analysis to the perceived space, the points belonging to the 2-Sphere defined by Equation (2.9) (with $\chi=\chi_{\max }$ ) are not influenced by the creation of the singularity: hence, their distance from the origin (as well as their radial extension) must be preserved.

Obviously, $R_{s}$ must represent an absolute maximum for $r$, which is clearly supposed to be monotonically increasing (in the range $0<\chi<\chi_{\max }$ ).

Lastly, since the one herein proposed is not a Pseudo-River Model [40], space does not flow towards the singularity: as a consequence, the angular distance between whatever couple of points must be considered as being constant.

In the light of the previous assumptions, resorting to the subscript " $g$ ", from now onwards, every time we refer to a quantity measured after the creation of the singularity, we have to simply impose the following [18]:

$$
R_{p, g}=\int_{0}^{\chi} \sqrt{\left(\frac{\mathrm{d} r}{\mathrm{~d} \chi}\right)^{2}+r^{2}} \mathrm{~d} \chi=R_{s} \chi=R_{p} .
$$

From the previous, if the derivative of $r$ with respect to $\chi$ is not null for $\chi<\chi_{\max }$ (otherwise we would banally have $r=R_{s}$ ), the underlying equation is easily obtained:

$$
\frac{\mathrm{d}^{2} r}{\mathrm{~d} \chi^{2}}+r=0
$$

As for the boundary conditions, according to our hypotheses, we have:

$$
r\left(\chi_{\max }\right)=R_{s},
$$




$$
\frac{\mathrm{d} r}{\mathrm{~d} \chi}\left(\chi_{\max }\right)=0 .
$$

From Equation (6.23), taking into account Equations (6.24) and (6.25), we obtain:

$$
\begin{aligned}
& r(\chi)=R_{s} \cos \left(\chi_{\max }-\chi\right), \\
& r_{\min }=r(0)=R_{s} \cos \chi_{\max } .
\end{aligned}
$$

Figure 8 qualitatively shows how space results in being deformed due to the Gravitational Singularity, perceived as being placed in $O_{g}$.

At the beginning, the origin coincides with $O$. If we concentrate in $O$ (actually along the segment bordered by $C$ and $O$ ) the mass of the Spherical Sector (actually a Hyper-Spherical Sector) with an amplitude equal to $2 \chi_{\max }$, space undergoes a contraction. The new origin coincides with $O_{g}$, and the surrounding space is symmetrically warped. The initial radial coordinate of a generic point $P$ (actually its initial radial extension) is represented by the segment bordered by $C$ and $P$. The corresponding angular distance is denoted by $\chi$. The final coordinate (actually the final radial extension), represented by the segment bordered by $C$ and $P_{g}$, is shorter than the initial one, and its value is provided by Equation (6.26). The proper radius does not undergo any modification: the arc bordered by $O$ and $P$, in fact, is evidently equal to the one bordered by $O_{g}$ and $P_{g}$.

If we denote with $x$ the Reduced Forecast Radius, we have:

$$
R_{c, g}=x=r \sin \chi=R_{s} \sin \chi \cos \left(\chi_{\max }-\chi\right) \text {. }
$$

Now, by virtue pf Equations (6.26) and (6.27) we can immediately write:

$$
\begin{gathered}
\delta(\chi)=R_{s}-r(\chi)=R_{s}\left[1-\cos \left(\chi_{\max }-\chi\right)\right], \\
\delta_{\max }=\delta(0)=R_{s}\left(1-\cos \chi_{\max }\right) .
\end{gathered}
$$

Let us now denote with $M_{\text {tot }}$ the total mass of the 3-Ball and with $M_{\chi_{\max }}$ the mass contained in the spherical sector characterized by an amplitude equal to $2 \chi_{\max }$.

By virtue of Equation (6.30), we can write (with obvious meaning of the notation):

$$
\begin{gathered}
\frac{M_{\chi_{\max }}}{M_{\text {tot }}}=1-\cos \chi_{\max }=\frac{\delta_{\max }}{R_{s}}, \\
\delta_{\max }=R_{s} \frac{M_{\chi_{\max }}}{M_{t o t}}=\frac{2 G M_{\chi_{\max }}}{c^{2}}=R_{s, \chi_{\max }} .
\end{gathered}
$$

In other terms, the procedure entails a reduction of the radial coordinate of $O$ (actually, the material segment bordered by $C$ and $O$ undergoes a contraction) the size of which is equal to the Schwarzschild Radius corresponding to $M_{\chi_{\max }}$.

Figure 9 shows once again (in detail) how the singularity, perceived as being placed in $O_{g}$, does not influence the measured distance (the proper radius). 


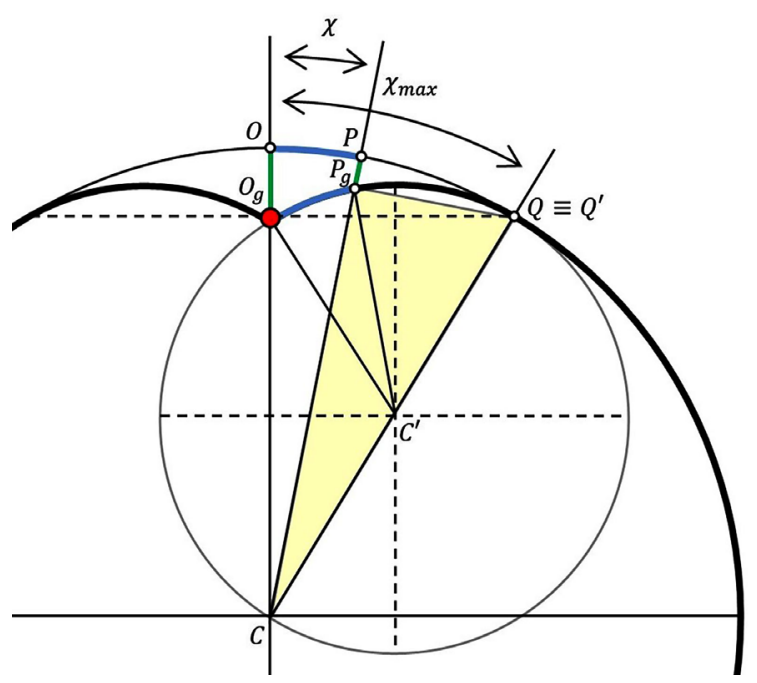

Figure 8. Gravitational singularity.

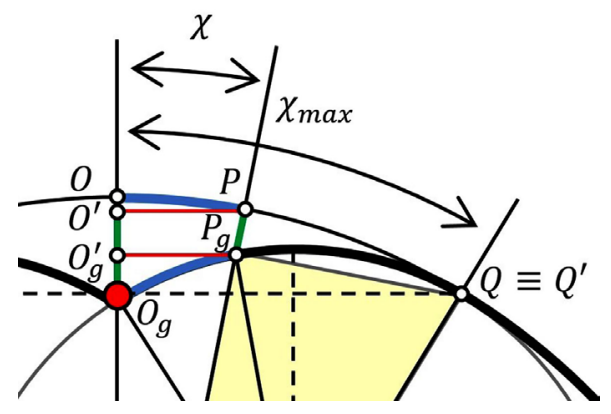

Figure 9. Gravitational singularity (particular).

The arc bordered by $O$ and $P$, is evidently equal to the one bordered by $O_{g}$ and $P_{g}$. On the contrary, the Forecast Radius undergoes a reduction. The segment bordered by $B$ and $P$ represents the Forecast Radius $(X)$ when matter is evenly spread; the segment bordered by $B_{g}$ and $P_{g}$ represents the Reduced Forecast Radius $(x)$.

\subsection{Quantization (Static Configuration)}

If mass homogeneously fills the 4-Ball with which we identify the Universe (static configuration), by virtue of the symmetry, the energy of a material segment provided with a mass $M$, can be written as in Equation (3.22).

The Linear Mass Density, which we keep on denoting with $\bar{M}$, can be immediately obtained from Equation (3.6) by banally replacing $R$ with $R_{s}$ :

$$
\bar{M}=\frac{M}{R_{s}} .
$$

By virtue of the foregoing, taking into account Equation (3.22), the (Linear) Energy Density can be defined as follows:

$$
\bar{E}=\frac{E}{R_{s}}=\frac{M c^{2}}{R_{s}}=\bar{M} c^{2} .
$$


Keeping on denoting with $\Delta R_{\min }$ the (Radial) Quantum of Space and with $m$ the punctual mass, Equations (3.47) and (3.48) can be immediately rewritten as follows:

$$
\begin{gathered}
R_{s}=\mathcal{N} \Delta R_{\min }, \\
m=\bar{M} \Delta R_{\min }=\frac{M}{R_{s}} \Delta R_{\min } .
\end{gathered}
$$

As for the energy of a punctual mass, denoted by $E_{m}$, from the Equations (6.34) and (6.36) we have:

$$
E_{m}=\bar{E} \Delta R_{\min }=\frac{M c^{2}}{R_{s}} \Delta R_{\min }=m c^{2} .
$$

Let us now introduce some Fundamental Quantities.

If we denote with $M_{\min }$ and $E_{\min }$, respectively, he Minimum Linear Mass and the corresponding energy, taking into account Equation (3.22) we can write:

$$
E_{\min }=M_{\min } c^{2} \text {. }
$$

By virtue of the foregoing, taking into account Equation (6.33), we define the Minimum Linear Mass Density, denoted by $\bar{M}_{\min }$, as follows:

$$
\bar{M}_{\min }=\frac{M_{\min }}{R_{s}} .
$$

Denoting with $\bar{E}_{\min }$ the Minimum (Linear) Energy Density, from Equations (6.34) and (6.38) we have:

$$
\bar{E}_{\min }=\frac{E_{\min }}{R_{s}}=\frac{M_{\min } c^{2}}{R_{s}}=\bar{M}_{\min } c^{2} .
$$

Denoting with $m_{\min }$ the Minimum Punctual Mass, from Equations (6.36) and (6.39) we immediately obtain:

$$
m_{\min }=\bar{M}_{\min } \Delta R_{\min }=\frac{M_{\min }}{R_{s}} \Delta R_{\min } .
$$

Consequently, denoting with $E_{m, \text { min }}$ the Minimum Energy for a Punctual Mass, from Equations (6.37), (6.40) and (6.41) we have:

$$
E_{m, \min }=\bar{E}_{\min } \Delta R_{\min }=\frac{M_{\min } c^{2}}{R_{s}} \Delta R_{\min }=m_{\min } c^{2} .
$$

By virtue of Equation (6.32), we can now write the expression for the Minimum Schwarzschild Radius.

$$
R_{s, \min }=\frac{2 G M_{\min }}{c^{2}} .
$$

We can interpret $M_{\min }$ as the value of linear mass, still unknown, below which no deformation of spatial lattice (no radial contraction) occurs.

Now, taking into account the central symmetry we have been resorting to in building our simple model, the Maximum Wavelength for a photon can be written as follows: 


$$
\lambda_{\max }=\pi R_{s} .
$$

Taking into account the foregoing equation, denoting with $h$, as usual, the Planck Constant, we can determine the Minimum (Perceived) Energy:

$$
E_{\text {photon, } \min }=\frac{h c}{\lambda_{\max }}=\frac{h c}{\pi R_{s}} .
$$

From the previous, taking into account Equation (6.42), we have:

$$
\begin{gathered}
E_{\text {photon, } \min }=\frac{h c}{\lambda_{\max }}=\frac{h c}{\pi R_{s}}=m_{\min } c^{2}, \\
m_{\min }=\frac{h}{\pi c R_{s}} .
\end{gathered}
$$

For a (linear) mass to induce a spatial deformation (a radial contraction), the value of the corresponding Schwarzschild Radius must be greater than or equal to the value of the (Radial) Quantum of Space.

Let us carry out the following position:

$$
\Delta R_{\min }=R_{s, \min } .
$$

Now, from Equations (6.41) and (6.43), taking into account the previous, we have:

$$
m_{\text {min }}=\frac{M_{\text {min }}}{R_{s}} \Delta R_{\min }=\frac{M_{\min }}{R_{s}} R_{s, \min }=\frac{2 G M_{\min }^{2}}{R_{s} c^{2}} .
$$

From the foregoing, by virtue of Equation (6.47), we obtain:

$$
\frac{2 G M_{\min }^{2}}{R_{s} c^{2}}=\frac{h}{\pi c R_{s}} .
$$

From the previous, taking into account the well-known definition of Reduced Planck Constant, we finally obtain:

$$
\begin{gathered}
M_{\min }^{2}=\left(\frac{h}{2 \pi}\right) \frac{c}{G}=\frac{\hbar c}{G}, \\
M_{\text {min }}=\sqrt{\frac{\hbar c}{G}}=M_{P} .
\end{gathered}
$$

The Minimum Value for the Linear Mass formally coincides with the one of the so-called Planck Mass, herein denoted with $M_{P}$.

From Equations (6.35) and (6.41), taking into account the previous, we have:

$$
m_{\text {min }}=\frac{\Delta R_{\min }}{R_{s}} M_{\text {min }}=\frac{1}{\mathcal{N}} M_{\text {min }}=\frac{1}{\mathcal{N}} \sqrt{\frac{\hbar c}{G}} .
$$

From Equations (6.43) and (6.52), resorting to the definition of Planck Length, we finally obtain the value of the (Radial) Quantum of Space:

$$
\Delta R_{\min }=R_{s, \min }=\frac{2 G M_{\min }}{c^{2}}=2 \sqrt{\frac{\hbar G}{c^{3}}}=2 \ell_{P} .
$$

The value just found is evidently coherent with the one in Equation (5.24). 
At this point, we can also carry out a Time Quantization. By virtue Equation (6.54), denoting with $t_{P}$ the so-called Planck Time, we define the Quantum of Time as follows:

$$
\Delta t_{\min }=\frac{\Delta R_{\min }}{c}=2 \sqrt{\frac{\hbar G}{c^{5}}}=2 t_{P} .
$$

\section{7. (Non-Rotating and Non-Charged) "Black Holes”}

Referring to Figure 8, let us now suppose that the total available mass may be concentrated in $O$ : in other terms, we set $\chi_{\max }=\pi / 2$.

Abiding by our model, from Equation (6.26) we obtain:

$$
\begin{gathered}
r(\chi)=R_{s} \cos \left(\frac{\pi}{2}-\chi\right)=R_{s} \sin \chi, \\
r_{\max }=r\left(\frac{\pi}{2}\right)=R_{s} .
\end{gathered}
$$

Evidently, the value of the Radial Coordinate (the Reduced Radial Extension) coincides, for any $\chi$, with the one of the Predicted Radius provided by Equation (6.5):

$$
R_{c}=X=r .
$$

For the Reduced Predicted Radius, by virtue of Equation (6.28), we have:

$$
R_{c, g}=x=X \sin \chi=R_{s} \sin ^{2} \chi=R_{c} \sin \chi .
$$

The scenario is qualitatively portrayed in Figure 10.

\subsection{Variable Quanta (And Locally Variable Planck "Constant")}

We want now to carry out a quantization of the coordinate $r$.

As shown in Equation (7.1), this coordinate exclusively depends on the angular distance $\chi$ (since we have set $\left.\chi_{\max }=\pi / 2\right)$ : the more we approach the "singularity", the more the value of $r$ decreases.

However, once again, $r$ does not shorten within space: it shortens together with space, since space itself undergoes a progressive (radial) contraction in approaching the "singularity" (actually, as we will later see, the previous statement is not properly correct).

Consequently, we consider a Variable (Radial) Space-Quantum, denoted with $\Delta r_{\min , \chi}$, the value of which depends on the angular distance $\chi$.

If $\mathcal{N}$ represents the same integer introduced in Equation (3.47), we impose:

$$
r=\mathcal{N} \Delta r_{\min , \chi} \text {. }
$$

According to the previous, taking into account Equations (6.35), (6.54) and (7.2), we must have, with obvious meaning of the notation, the following:

$$
\begin{gathered}
r_{\text {max }}=\mathcal{N} \Delta r_{\min , \pi / 2}=R_{s}=\mathcal{N} \Delta R_{\min }, \\
\Delta r_{\min , \pi / 2}=\Delta R_{\min }=2 \ell_{P} .
\end{gathered}
$$

From Equations (7.5) and (7.6) we immediately obtain: 


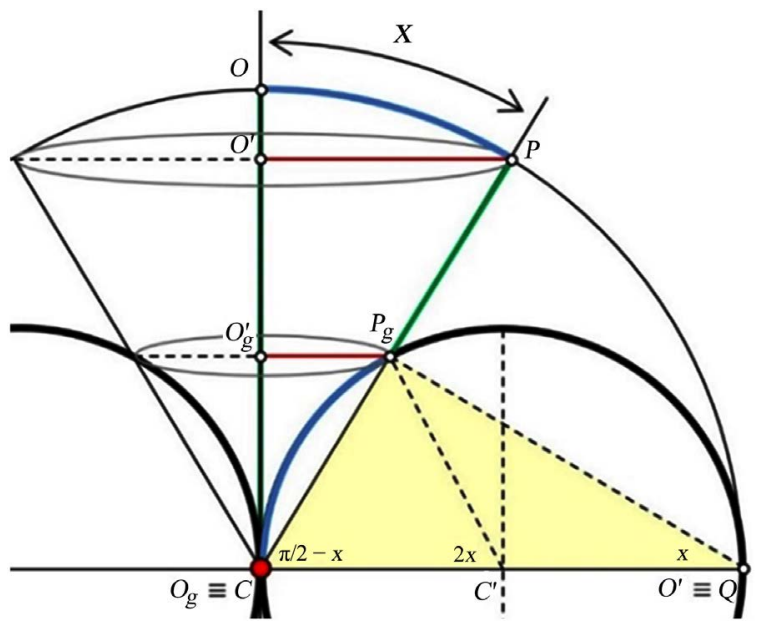

Figure 10. "Black hole".

$$
\mathcal{N}=\frac{r}{\Delta r_{\min , \chi}}=\frac{R_{s}}{\Delta r_{\min , \pi / 2}}=\frac{R_{s}}{\Delta R_{\min }} .
$$

From Equation (7.8), taking into account Equation (7.1), we have:

$$
\frac{\Delta r_{\min , \chi}}{\Delta r_{\min , \pi / 2}}=\frac{\Delta r_{\min , \chi}}{\Delta R_{\min }}=\frac{r}{R_{s}}=\sin \chi .
$$

In the light of the previous relation, we can now introduce the following Non-Dimensional Parameter, which represents nothing but a simple Scale Factor.

$$
\eta_{\Delta r_{\min }}=\frac{\Delta r_{\min , \pi / 2}}{\Delta r_{\min , \chi}}=\frac{\Delta R_{\min }}{\Delta r_{\min , \chi}}=\frac{R_{s}}{r}=\frac{1}{\sin \chi} .
$$

Now, from Equations (6.54) and (7.9) we immediately obtain:

$$
\Delta r_{\min , \chi}=\sin \chi \Delta R_{\min }=2 \ell_{P} \sin \chi=2 \sqrt{\frac{\left(\hbar \sin ^{2} \chi\right) G}{c^{3}}} .
$$

Let us carry out the underlying position:

$$
\hbar_{\chi}=\hbar \sin ^{2} \chi
$$

In other terms, we have been hypothesizing a local variability of the Planck “Constant" [41] [42] [43] [44].

From the previous, taking into account Equation (7.9), we deduce, with obvious meaning of the notation, the following:

$$
\frac{h_{\chi}}{h}=\frac{h_{\chi}}{h_{\pi / 2}}=\sin ^{2} \chi=\left(\frac{r}{R_{s}}\right)^{2} .
$$

Finally, from Equation (7.11) and (7.11), denoting with $\ell_{P, \chi}$ the Variable (Reduced) Planck Length, we immediately obtain:

$$
\Delta r_{\min , \chi}=2 \sqrt{\frac{\hbar_{\chi} G}{c^{3}}}=2 \ell_{P, \chi} .
$$


By virtue of the previous, the Variable Quantum of Time is now defined as follows:

$$
\Delta t_{\min , g}(\chi)=\Delta t_{\min , \chi}=\frac{\Delta r_{\min , \chi}}{c}=2 \sqrt{\frac{\hbar}{c^{5}}}=2 t_{P, \chi} .
$$

From the foregoing, taking into account Equations (6.55) and (7.12), we obtain:

$$
\Delta t_{\min , \pi / 2}=\Delta t_{\min }=2 t_{P}
$$

Finally, from Equations (6.55), (7.13) and (7.16), we easily deduce:

$$
\frac{\Delta t_{\min , \chi}}{\Delta t_{\min , \pi / 2}}=\frac{\Delta t_{\min , \chi}}{\Delta t_{\min }}=\sin \chi=\frac{1}{\eta_{\Delta r_{\min }}} .
$$

\section{2. “Gravitational” Mass}

In case of singularity, a material segment does not undergo any radial reduction (in other terms, it does not shorten within space).

As previously remarked, in fact, both the segment and the corresponding space undergo a radial contraction (the segment shortens together with space).

Consequently, the mass of any material segment must be regarded as constant.

If we now denote with $M$ the (constant) mass of a "Test" Material Segment, the (Variable) Linear Mass Density, in case of singularity, can be defined as follows:

$$
\bar{M}=\frac{M}{r} .
$$

The relation that express the Mass of a Test Particle (the mass we perceive) must clearly resemble Equation (6.36).

Consequently, taking into account Equations (7.9) and (7.18), we can write, with obvious meaning of the notation, the following:

$$
m_{\chi}=\bar{M} \Delta r_{\min , \chi}=M \frac{\Delta r_{\min , \chi}}{r}=M \frac{\Delta r_{\min , \pi / 2}}{R_{s}}=m_{\pi / 2} .
$$

From the previous, by virtue of Equations (6.36) and (7.6), we have:

$$
m_{\chi}=m_{\pi / 2}=\frac{M}{R_{s}} \Delta R_{\min }=\frac{M}{\mathcal{N}}=m
$$

In other terms, thanks to the position in Equation (7.5) (the meaning of which should now be clearer), the "Gravitational" Mass and the Inertial Mass coincide (as requested by the Equivalence Principle) [3] [4].

\subsection{The Conservation of Energy (Motion Induced by a "Singularity")}

In presence of a singularity, the total energy of a material segment cannot be written by directly resorting to Equation (3.30). 
Due to the singularity, in fact, the linear density, defined in Equation (7.18), is no longer constant (once again, the segment does not shorten within space, but together with space): consequently, Equations (3.7) and (3.8) are no longer valid. In particular, since the linear mass is now considered as being constant, the term in Equation (3.28) must necessarily vanish.

Therefore, the conservation of energy can still be expressed by Equation (3.30), on condition that we preliminarily set $M^{\prime}=M$ and replace, obviously, $R^{\prime}$ with $r$.

Finally, we have:

$$
E=M c^{2}=M v^{2}+\left(\frac{r}{R_{s}}\right)^{2} M c^{2} .
$$

From the previous relation, taking into account Equation (7.20), being $\mathcal{N} \neq 0$, we immediately obtain the conservation of energy for a (Free-Falling) Test Particle:

$$
\begin{gathered}
E_{m}=\frac{E}{\mathcal{N}}=\frac{M}{\mathcal{N}} c^{2}=\frac{M}{\mathcal{N}} v^{2}+\left(\frac{r}{R_{s}}\right)^{2} \frac{M}{\mathcal{N}} c^{2}, \\
E_{m}=m c^{2}=m v^{2}+\left(\frac{r}{R_{s}}\right)^{2} m c^{2} .
\end{gathered}
$$

According to the foregoing equation, referring to Figure 10, the test particle placed in $P_{g}$, in order to preserve its energy, starts moving towards $C$ (actually, the segment bordered by $C$ and $P_{g}$ starts rotating counter-clockwise).

\subsection{The (Gravitational) Potential and the Coordinate $\boldsymbol{R}^{*}$}

From Equations (7.1) and (7.23) we can easily deduce as follows:

$$
\begin{gathered}
v=c \sqrt{1-\left(\frac{r}{R_{s}}\right)^{2}}=c \cos \chi, \\
\frac{r}{R_{s}}=\sqrt{1-\left(\frac{v}{c}\right)^{2}}=\sin \chi, \\
\frac{1}{2} m v^{2}-\frac{1}{2} m c^{2}\left[1-\left(\frac{r}{R_{s}}\right)^{2}\right]=\frac{1}{2} m v^{2}-\frac{1}{2} m c^{2} \cos ^{2} \chi=0 .
\end{gathered}
$$

From Equation (6.1) we immediately obtain:

$$
c^{2}=\frac{2 G M_{t o t}}{R_{s}} .
$$

Consequently, we have:

$$
-\frac{1}{2} c^{2} \cos ^{2} \chi=-\frac{G M_{t o t}}{R_{s}} \cos ^{2} \chi .
$$

Let us introduce a New Coordinate [18], denoted by $R^{*}$, defined as follows: 


$$
R^{*}(\chi)=\frac{R_{s}}{\cos ^{2} \chi}
$$

Obviously, from the foregoing we have:

$$
\begin{gathered}
R^{*}(0)=R_{s}, \\
\lim _{\chi \rightarrow \pi / 2} R^{*}=+\infty, \\
\cos \chi=\sqrt{\frac{R_{s}}{R^{*}}}, \\
\sin \chi=\sqrt{1-\frac{R_{s}}{R^{*}}} .
\end{gathered}
$$

Now, from Equation (7.26), taking into account Equation (7.28), we obtain:

$$
\frac{1}{2} m v^{2}-\frac{G M_{t o t}}{R^{*}} m=0 .
$$

Let us define the Pseudo-Newtonian Potential, denoted by $\phi$, as follows:

$$
-\frac{G M_{t o t}}{R^{*}}=-\frac{1}{2} c^{2} \cos ^{2} \chi=\phi(\chi) .
$$

Evidently, the potential reaches its minimum at $\chi=0$ (or, equivalently, at $\left.R^{*}=R_{s}\right)$.

Therefore, we can write:

$$
\phi(0)=\phi_{\min }=-\frac{1}{2} c^{2} .
$$

Finally, from Equation (7.26), taking into account Equation (7.35), we have:

$$
\frac{1}{2} m v^{2}+\phi m=0
$$

The previous equation represents, net of the symmetry, the conservation of energy for a Free-Falling Particle (the Free-Fall starts from rest at $\chi=\pi / 2$ ) [3].

\subsection{Speed of a Free-Falling (Test) Particle}

From Equations (7.24), (7.32) and (7.35), we have:

$$
v=c \cos \chi=\sqrt{\frac{2 G M_{t o t}}{R^{*}}}=c \sqrt{\frac{2 G M_{t o t}}{c^{2} R^{*}}}=c \sqrt{\frac{R_{s}}{R^{*}}}=\sqrt{2 \phi} .
$$

According to the Schwarzschild Geometry (see Paragraph 8), the value just deduced formally coincides with the (escape) speed as measured by a so-called Shell Observer (a stationary observer placed at a finite distance $R^{*}$ from the singularity) [3].

As for the Velocity Components, denoted by $v_{I}$ and $v_{I I}$, we can evidently write:

$$
v=\sqrt{v_{I}^{2}+v_{I I}^{2}}
$$

Now, from Equation (7.38) we immediately obtain: 


$$
\frac{R_{s}}{R^{*}}=\frac{2 \phi}{c^{2}} \text {. }
$$

Consequently, $v_{I}$ and $v_{I I}$ assume the following forms:

$$
\begin{gathered}
v_{I}=c \sin \chi \cos \chi=c \sqrt{\left(1-\frac{R_{s}}{R^{*}}\right) \frac{R_{s}}{R^{*}}}=c \sqrt{\frac{2 \phi}{c^{2}}-\left(\frac{2 \phi}{c^{2}}\right)^{2}}, \\
v_{I I}=c \cos ^{2}=c \frac{R_{s}}{R^{*}}=\frac{2 \phi}{c} .
\end{gathered}
$$

The Velocity Components are depicted in Figure 11.

\subsection{Parameterization}

We want to find two new coordinates, denoted with $R_{s}$ and $r_{K}^{*}$, which could "play the role", respectively, of $R_{s}$ and $r$. Let us denote with $R_{p}^{*}$ the Parameterized Predicted Radius, and with $R_{p, g}^{*}$ the Parameterized Reduced Predicted Radius.

Firstly, in the light of Equation (6.22), we must impose:

$$
R_{p, g}^{*}=\int_{0}^{\chi} \sqrt{\left(\frac{\mathrm{d} r_{K}^{*}}{\mathrm{~d} \chi}\right)^{2}+r_{K}^{* 2}} \mathrm{~d} \chi=\int_{0}^{\chi} \sqrt{\left(\frac{\mathrm{d} R_{K}^{*}}{\mathrm{~d} \chi}\right)^{2}+R_{K}^{*}} \mathrm{~d} \chi=R_{p}^{*} .
$$

Secondly, in the light of Equation (7.1), we must additionally impose:

$$
r_{K}^{*}=R_{K}^{*} \sin \chi .
$$

From the two previous equations, if the derivative of $R_{K}^{*}$ with respect to $\chi$ does not vanish (for $\chi>0$ ), we easily obtain the following:

$$
\frac{\mathrm{d} R_{K}^{*}}{R_{K}^{*}}=2 \tan \chi \mathrm{d} \chi
$$

The general solution of the foregoing, denoting with $K$ an arbitrary constant, is:

$$
R_{K}^{*}=\frac{K}{\cos ^{2} \chi} .
$$

From the previous we immediately deduce the underlying noteworthy identities:

$$
\begin{gathered}
\cos \chi=\sqrt{\frac{K}{R^{*}}}, \\
\sin \chi=\sqrt{1-\frac{K}{R_{K}^{*}}} .
\end{gathered}
$$

From Equation (7.46) we have:

$$
\frac{\mathrm{d} R_{K}^{*}}{\mathrm{~d} \chi}=2 K \frac{\sin \chi}{\cos ^{3} \chi} .
$$

From Equations (7.44) and (7.46) we have:

$$
r_{K}^{*}=R_{K}^{*} \sin \chi=K \frac{\sin \chi}{\cos ^{2} \chi},
$$




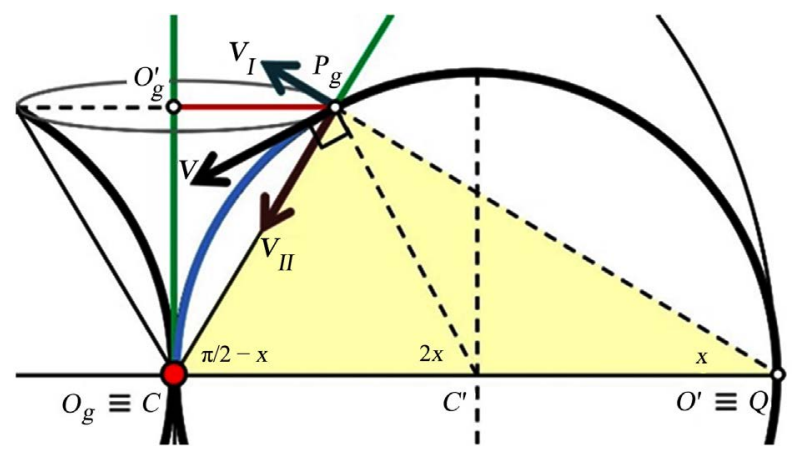

Figure 11. Velocity components.

$$
\frac{\mathrm{d} r_{K}^{*}}{\mathrm{~d} \chi}=K \frac{1+\sin ^{2} \chi}{\cos ^{3} \chi} .
$$

As for the parameterized predicted radius, denoted by $R_{c}^{*}$ or $X_{K}^{*}$, coherently with Equations (7.1) and (7.3), we have:

$$
R_{c}^{*}=X_{K}^{*}=R_{K}^{*} \sin \chi=r_{K}^{*} .
$$

According to Equation (7.4), the relation between parameterized predicted radius (no singularity) and parameterized reduced predicted radius (in case singularity, additional subscript " $g$ ") must be the following:

$$
R_{c, g}^{*}=R_{c}^{*} \sin \chi
$$

Therefore, by virtue of Equations (7.52) and (7.53), for the parameterized reduced predicted radius, equivalently denoted by $x_{K}^{*}$, we have:

$$
R_{c, g}^{*}=x_{K}^{*}=R_{c}^{*} \sin \chi=X_{K}^{*} \sin \chi=r_{K}^{*} \sin \chi=R_{K}^{*} \sin ^{2} \chi .
$$

From the previous, taking into account Equation (7.47), we obtain:

$$
\begin{gathered}
x_{K}^{*}=K \tan ^{2} \chi=\frac{K}{\cos ^{2} \chi}-K=R_{K}^{*}-K, \\
\frac{\mathrm{d} x_{K}^{*}}{\mathrm{~d} \chi}=\frac{\mathrm{d} R_{K}^{*}}{\mathrm{~d} \chi} .
\end{gathered}
$$

According to Equation (7.43), the parameterized proper radius is not influenced by the singularity. Therefore, from Equations (7.46), (7.49), (7.50) and (7.51), we have:

$$
\begin{aligned}
& \mathrm{d} R_{p, g}^{*}=\sqrt{\left(\frac{\mathrm{d} R_{K}^{*}}{\mathrm{~d} \chi}\right)^{2}+R_{K}^{* 2} \mathrm{~d} \chi}=\frac{\mathrm{d} R_{K}^{*}}{\mathrm{~d} \chi} \sqrt{1+\frac{1}{4 \tan ^{2} \chi}} \mathrm{d} \chi \\
& =\sqrt{1+\frac{1}{4 \tan ^{2} \chi}} \mathrm{d} R_{K}^{*}=\mathrm{d} R_{p}^{*}, \\
& \mathrm{~d} R_{p, g}^{*}=\sqrt{\left(\frac{\mathrm{d} r_{K}^{*}}{\mathrm{~d} \chi}\right)^{2}+r_{K}^{* 2}} \mathrm{~d} \chi=\frac{\mathrm{d} r_{K}^{*}}{\mathrm{~d} \chi} \sqrt{1+\left(\frac{\sin \chi \cos \chi}{1+\sin ^{2} \chi}\right)^{2}} \mathrm{~d} \chi \\
& =\sqrt{1+\frac{\sin ^{2} \chi \cos ^{2} \chi}{\left(1+\sin ^{2} \chi\right)^{2}}} \mathrm{~d} r_{K}^{*}=\mathrm{d} R_{p}^{*},
\end{aligned}
$$




$$
\mathrm{d} R_{p, g}^{*}=\mathrm{d} R_{p}^{*}=\sqrt{1+\frac{1}{4 \tan ^{2} \chi}} \mathrm{d} R_{K}^{*}=\sqrt{1+\frac{\sin ^{2} \chi \cos ^{2} \chi}{\left(1+\sin ^{2} \chi\right)^{2}}} \mathrm{~d} r_{K}^{*} .
$$

In Figure 12, a useful comparison between old and new (parameterized) coordinates (in the particular case $K=R_{s}$ ) is qualitatively displayed.

\subsection{Parameterized Quantization}

The parameterization also affects the quantization [18].

Obviously, it is not a real phenomenon.

Coherently with the parameterization we have been resorting to, taking into account Equations (6.35) and (7.7), we must now impose, with obvious meaning of the notation, the following:

$$
R_{K}^{*}=\mathcal{N} \Delta R_{\min }^{*}=\mathcal{N} \Delta r_{\min , \pi / 2}^{*}
$$

From the previous, by virtue of Equation (7.46), we obtain:

$$
\Delta R_{\min }^{*}=\frac{R_{K}^{*}}{\mathcal{N}}=\frac{K}{\mathcal{N}} \frac{1}{\cos ^{2} \chi} .
$$

If we set $K=R_{s}$, taking into account Equations (6.35), from the foregoing we have:

$$
\Delta R_{\min }^{*}=\frac{R_{s}}{\mathcal{N}} \frac{1}{\cos ^{2} \chi}=\frac{\Delta R_{\min }}{\cos ^{2} \chi} .
$$

Obviously, by virtue of Equation (7.5), we must also impose:

$$
r_{K}^{*}=\mathcal{N} \Delta r_{\min , \chi}^{*} .
$$

From the previous, by virtue of Equation (7.50), we obtain:

$$
\Delta r_{\min , \chi}^{*}=\frac{r_{K}^{*}}{\mathcal{N}}=\frac{K}{\mathcal{N}} \frac{\sin \chi}{\cos ^{2} \chi} .
$$

If we set $K=R_{s}$, taking into account Equation (7.9), from the foregoing we have:

$$
\Delta r_{\min }^{*}=\frac{R_{s} \sin \chi}{\mathcal{N}} \frac{1}{\cos ^{2} \chi}=\frac{\Delta R_{\min } \sin \chi}{\cos ^{2} \chi}=\frac{\Delta r_{\min , \chi}}{\cos ^{2} \chi} .
$$
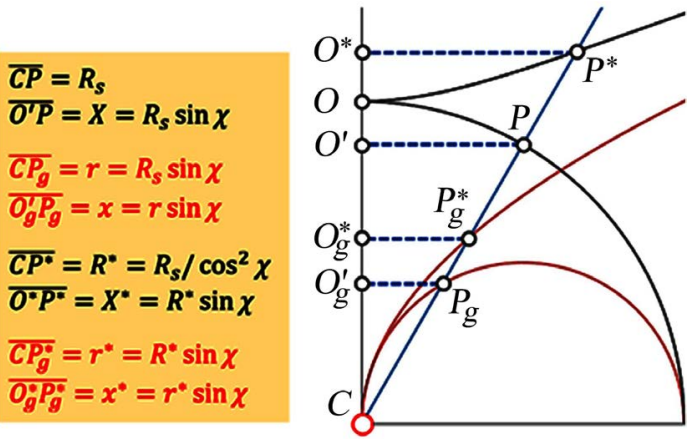

Figure 12. Parameterization $\left(K=R_{s}\right)$. 
Evidently, by virtue of Equations (7.60) and (7.63), we can write:

$$
\frac{\Delta r_{\min , \chi}^{*}}{\Delta R_{\min }^{*}}=\sin \chi \text {. }
$$

Therefore, at infinity $(\chi \rightarrow \pi / 2)$, we obtain:

$$
\Delta r_{\min , \pi / 2}^{*}=\Delta R_{\min }^{*} \text {. }
$$

From Equations (7.9), (7.60) and (7.63), taking into account the foregoing, we have:

$$
\frac{\Delta r_{\min , \chi}^{*}}{\Delta r_{\min , \pi / 2}^{*}}=\frac{\Delta r_{\min , \chi}^{*}}{\Delta R_{\min }^{*}}=\sin \chi=\frac{\Delta r_{\min , \chi}}{\Delta R_{\min }}=\frac{\Delta r_{\min , \chi}}{\Delta r_{\min , \pi / 2}} .
$$

In the light of the previous, resorting to Equation (7.48), we can now introduce the new following Parameterized Scale Factor.

$$
\eta_{\Delta r_{\min }}^{*}=\frac{\Delta R_{\min }^{*}}{\Delta r_{\min , \chi}^{*}}=\frac{\Delta R_{\min }}{\Delta r_{\min , \chi}}=\frac{1}{\sin \chi}=\frac{1}{\sqrt{1-\frac{K}{R_{K}^{*}}}} .
$$

The Parameterized Quantum of Time is defined as follows:

$$
\Delta t_{\min , g}^{*}(\chi)=\Delta t_{\min , \chi}^{*}=\frac{\Delta r_{\min , \chi}^{*}}{c} .
$$

Taking into account Equations (7.17) and (7.68), from the previous we obtain:

$$
\begin{gathered}
\frac{\Delta t_{\min , \chi}^{*}}{\Delta t_{\min , \pi / 2}^{*}}=\frac{\Delta r_{\min , \chi}^{*}}{\Delta R_{\min }^{*}}=\sin \chi=\frac{1}{\eta_{\Delta r_{\min }}^{*}}=\sqrt{1-\frac{K}{R_{K}^{*}}}, \\
\frac{\Delta t_{\min , \chi}^{*}}{\Delta t_{\min , \pi / 2}^{*}}=\frac{\Delta t_{\min , \chi}^{*}}{\Delta t_{\min }^{*}}=\frac{\Delta t_{\min , \chi}}{\Delta t_{\min }}=\frac{\Delta t_{\min , \chi}}{\Delta t_{\min , \pi / 2}} .
\end{gathered}
$$

It is worth highlighting how, from Equations (3.32), (7.10), (7.24), (7.47), (7.48) and (7.68), we easily obtain:

$$
\eta_{\Delta r_{\text {min }}}^{*}=\eta_{\Delta r_{\text {min }}}=\frac{1}{\sin \chi}=\frac{1}{\sqrt{1-\frac{K}{R_{K}^{*}}}}=\frac{1}{\sqrt{1-\left(\frac{v}{c}\right)^{2}}}=\gamma
$$

\section{Schwarzschild-Like Solution}

In 1916, Karl Schwarzschild succeeded in finding an exact solution to the Einstein Field Equations. The astonishing outcome was obtained by hypothesizing a spherically symmetric static scenario (all the time derivatives vanish), and imposing that, outside the mass that produces the field (the singularity is not considered as being part of the solution), there is the "absolute nothing" (neither matter nor energy).

In this section we firstly build an initial metric which, far from the origin (in correspondence of which the singularity will be later placed), may be considered as being flat. 
Then, we carry out two different derivations of the Schwarzschild-Like Solution [18].

The first procedure can be regarded as a conventional "mixed" derivation: conventional, since we clearly resort to GR (following, in a certain sense, a "Hilbert-Like" approach to the problem) [45], and "mixed", since we obtain the desired outcome by starting from a metric explicitly built as a function of the parametric coordinate $R_{K}^{*}$, defined in Equation (7.46) (obviously, this is not a peremptory condition, otherwise Schwarzschild himself could have never achieved his well-known solution).

The second (alternative) procedure, carried out without resorting to GR, is entirely based on a simple assumption (see Sub-Paragraph 8.3), which can be qualitatively and briefly summarized as follows: the "shape" of the Universe cannot be modified with respect to something else, taken as a reference.

\subsection{Initial Flat Metric (No Singularity)}

We can start from the following general metric:

$$
\mathrm{d} s^{* 2}=c^{2} \mathrm{~d} t^{* 2}-\mathrm{d} R_{p}^{* 2}-R_{c}^{* 2}\left(\mathrm{~d} \theta^{2}+\sin ^{2} \theta \mathrm{d} \varphi^{2}\right) .
$$

Now, from Equation (7.52) we have:

$$
\lim _{\chi \rightarrow \pi / 2} \frac{R_{c}^{*}}{R_{K}^{*}}=\lim _{\chi \rightarrow \pi / 2} \frac{X_{K}^{*}}{R_{K}^{*}}=\lim _{\chi \rightarrow \pi / 2} \frac{r_{K}^{*}}{R_{K}^{*}}=\lim _{\chi \rightarrow \pi / 2} \sin \chi=1 .
$$

Consequently, far from the origin, the parameterized predicted radius and the parameterized radial coordinate are interchangeable. We can write:

$$
R_{c}^{*} \cong R_{K}^{*} \text {. }
$$

Now, we evidently have:

$$
\lim _{\chi \rightarrow \pi / 2} \sqrt{1+\frac{1}{4 \tan ^{2} \chi}}=1 .
$$

Far from the origin, therefore, by virtue of Equation (7.56), the parameterized proper radius and the parameterized radial coordinate are interchangeable. We can write:

$$
\mathrm{d} R_{p}^{*} \cong \mathrm{d} R_{K}^{*} .
$$

Finally, far from the origin (in correspondence of which the singularity will be created), by virtue of Equations (8.3) and (8.5), from Equation (8.1) the following Flat Metric is immediately obtained:

$$
\mathrm{d} s^{* 2}=c^{2} \mathrm{~d} t^{* 2}-\mathrm{d} R_{K}^{* 2}-R_{K}^{* 2}\left(\mathrm{~d} \theta^{2}+\sin ^{2} \theta \mathrm{d} \varphi^{2}\right) .
$$

It is fundamental to underline how the approximation in Equation (8.3) prevents the parameterized predicted radius from assuming a null value. In detail, by virtue of Equation (7.46) ( $K \neq 0)$, with obvious meaning of the notation, we have:

$$
R_{c, \min }^{*}(\chi)=R_{c}^{*}(0)=R_{K}^{*}(0)=K
$$




\subsection{Schwarzschild-Like Solution: Conventional "Mixed" Derivation}

As is well known, the general static, spherically (and time) symmetric solution can be written as follows:

$$
\begin{aligned}
& \mathrm{d} s^{2}=A\left(R_{K}^{*}\right) c^{2} \mathrm{~d} t^{* 2}-B\left(R_{K}^{*}\right) \mathrm{d} R_{K}^{* 2}-C\left(R_{K}^{*}\right)\left(\mathrm{d} \theta^{2}+\sin ^{2} \theta \mathrm{d} \varphi^{2}\right), \\
& A\left(R_{K}^{*}\right), B\left(R_{K}^{*}\right), C\left(R_{K}^{*}\right)>0 .
\end{aligned}
$$

Let us carry out the following position [18]:

$$
\sqrt{C\left(R_{K}^{*}\right)}=R_{K}^{*} .
$$

Thanks to the previous, the metric in Equation (8.8) can be written as follows:

$$
\begin{aligned}
& \mathrm{d} s^{* 2}=A^{*}\left(R_{K}^{*}\right) c^{2} \mathrm{~d} t^{* 2}-B^{*}\left(R_{K}^{*}\right) \mathrm{d} R_{K}^{* 2}-R_{K}^{* 2}\left(\mathrm{~d} \theta^{2}+\sin ^{2} \theta \mathrm{d} \varphi^{2}\right), \\
& A^{*}\left(R_{K}^{*}\right), B^{*}\left(R_{K}^{*}\right)>0 .
\end{aligned}
$$

As for the Metric Tensor, from the foregoing we have:

$$
\begin{aligned}
g_{i j} & =\left[\begin{array}{cccc}
A^{*}\left(R_{K}^{*}\right) & 0 & 0 & 0 \\
0 & -B^{*}\left(R_{K}^{*}\right) & 0 & 0 \\
0 & 0 & -R_{K}^{* 2} & 0 \\
0 & 0 & 0 & -R_{K}^{* 2} \sin ^{2} \theta
\end{array}\right], \\
g^{i j} & =\left[\begin{array}{cccc}
\frac{1}{A^{*}\left(R_{K}^{*}\right)} & 0 & 0 & 0 \\
0 & -\frac{1}{B^{*}\left(R_{K}^{*}\right)} & 0 & 0 \\
0 & 0 & -\frac{1}{R_{K}^{* 2}} & 0 \\
0 & 0 & 0 & -\frac{1}{R_{K}^{* 2} \sin ^{2} \theta}
\end{array}\right] .
\end{aligned}
$$

It is fundamental to underline how $A^{*}$ and $B^{*}$ exclusively depend on the Flat Coordinate $R_{K}^{*}$, the form of which does not necessarily coincide with the one derived in equation (7.46). In particular, coherently with the hypothesized static scenario, whatever time derivatives must necessarily vanish.

Let us deduce the Christoffel Symbols. Generally, we have:

$$
\Gamma_{i j}^{k}=\frac{1}{2} g^{k h}\left(\frac{\partial g_{h i}}{\partial x^{j}}+\frac{\partial g_{h j}}{\partial x^{i}}-\frac{\partial g_{i j}}{\partial x^{h}}\right) \text {. }
$$

The indexes run from 0 to 3 ( 0 stands for $t^{*}, 1$ for $R_{K}^{*}, 2$ for $\theta$, and 3 for $\varphi)$.

Setting $k=0$, from Equations (8.11), (8.12) and (8.13), we obtain:

$$
\Gamma_{01}^{0}=\Gamma_{10}^{0}=\frac{1}{2 A^{*}} \frac{\mathrm{d} A^{*}}{\mathrm{~d} R_{K}^{*}} .
$$

All the other symbols (if $k=0$ ) vanish. 
Setting $k=1$, from Equations (8.11), (8.12) and (8.13), we obtain:

$$
\begin{gathered}
\Gamma_{00}^{1}=\frac{1}{2 B^{*}} \frac{\mathrm{d} A^{*}}{\mathrm{~d} R_{K}^{*}} ; \\
\Gamma_{11}^{1}=\frac{1}{2 B^{*}} \frac{\mathrm{d} B^{*}}{\mathrm{~d} R_{K}^{*}}, \Gamma_{12}^{1}=-\frac{R_{K}^{*}}{B^{*}}, \Gamma_{13}^{1}=-\frac{R_{K}^{*}}{B^{*}} \sin ^{2} \theta .
\end{gathered}
$$

All the other symbols (if $k=1$ ) vanish.

Setting $k=2$, from Equations (8.11), (8.12) and (8.13), we obtain:

$$
\begin{gathered}
\Gamma_{12}^{2}=\Gamma_{21}^{2}=\frac{1}{R_{K}^{*}}, \\
\Gamma_{33}^{2}=-\sin \theta \cos \theta .
\end{gathered}
$$

All the other symbols (if $k=2$ ) vanish.

Setting $k=3$, from Equations (8.11), (8.12) and (8.13), we obtain:

$$
\begin{gathered}
\Gamma_{13}^{3}=\Gamma_{31}^{3}=\frac{1}{R_{K}^{*}}, \\
\Gamma_{23}^{3}=\Gamma_{32}^{3}=\frac{1}{\tan \theta} .
\end{gathered}
$$

All the other symbols (if $k=3$ ) vanish.

Let's now deduce the components of the Ricci Tensor [3] [4]. Generally, with obvious meaning of the notation, we have:

$$
R i c_{i j}=\frac{\partial \Gamma_{i k}^{k}}{\partial x^{j}}-\frac{\partial \Gamma_{i j}^{k}}{\partial x^{k}}+\Gamma_{i k}^{l} \Gamma_{j l}^{k}-\Gamma_{i j}^{l} \Gamma_{k l}^{k} .
$$

From the previous, taking into account Equations from (8.14) to (8.20), the following non-vanishing components can be easily obtained:

$$
\begin{array}{r}
R i c_{00}=-\frac{1}{2 B^{*}} \frac{\mathrm{d}^{2} A^{*}}{\mathrm{~d} R_{K}^{* 2}}+\frac{1}{4 B^{*}} \frac{\mathrm{d} A^{*}}{\mathrm{~d} R_{K}^{*}}\left(\frac{1}{A^{*}} \frac{\mathrm{d} A^{*}}{\mathrm{~d} R_{K}^{*}}+\frac{1}{B^{*}} \frac{\mathrm{d} B^{*}}{\mathrm{~d} R_{K}^{*}}\right)-\frac{1}{R_{K}^{*} B^{*}} \frac{\mathrm{d} A^{*}}{\mathrm{~d} R_{K}^{*}}, \\
R i c_{11}=\frac{1}{2 A^{*}} \frac{\mathrm{d}^{2} A^{*}}{\mathrm{~d} R_{K}^{* 2}}-\frac{1}{4 A^{*}} \frac{\mathrm{d} A^{*}}{\mathrm{~d} R_{K}^{*}}\left(\frac{1}{A^{*}} \frac{\mathrm{d} A^{*}}{\mathrm{~d} R_{K}^{*}}+\frac{1}{B^{*}} \frac{\mathrm{d} B^{*}}{\mathrm{~d} R_{K}^{*}}\right)-\frac{1}{R_{K}^{*} B^{*}} \frac{\mathrm{d} B^{*}}{\mathrm{~d} R_{K}^{*}}, \\
R i c_{22}=\frac{1}{B^{*}}+\frac{R_{K}^{*}}{2 B^{*}}\left(\frac{1}{A^{*}} \frac{\mathrm{d} A^{*}}{\mathrm{~d} R_{K}^{*}}-\frac{1}{B^{*}} \frac{\mathrm{d} B^{*}}{\mathrm{~d} R_{K}^{*}}\right)-1, \\
R i c_{33}=\sin ^{2} \theta\left[\frac{1}{B^{*}}+\frac{R_{K}^{*}}{2 B^{*}}\left(\frac{1}{A^{*}} \frac{\mathrm{d} A^{*}}{\mathrm{~d} R_{K}^{*}}-\frac{1}{B^{*}} \frac{\mathrm{d} B^{*}}{\mathrm{~d} R_{K}^{*}}\right)-1\right]=\sin ^{2} \theta R_{22} .
\end{array}
$$

If we denote with $S_{R}$ the Ricci Scalar and with $T_{i j}$ the generic component of the Stress-Energy Tensor, the Einstein Field Equations [3] [4] can be written as follows:

$$
\text { Ric }_{i j}-\frac{1}{2} S_{R} g_{i j}=\frac{8 \pi G}{c^{4}} T_{i j} .
$$

We impose that, outside the mass that produces the field, there is neither 
matter nor energy $\left(T_{i j}=0\right)$ : consequently, the first member of Equation (8.26) (the so-called Einstein Tensor), must necessarily vanish. In other terms, we have:

$$
\operatorname{Ric}_{i j}-\frac{1}{2} S_{R} g_{i j}=0
$$

From the foregoing, exploiting the fact that the Einstein Tensor and the Ricci Tensor are trace-reverse of each other, we have:

$$
\operatorname{Ric}_{i j}=0 \text {. }
$$

Now, from Equations (8.22), (8.23) and (8.28) we obtain:

$$
\begin{gathered}
-\frac{1}{2 A^{*} B^{*}} \frac{\mathrm{d}^{2} A^{*}}{\mathrm{~d} R_{K}^{* 2}}+\frac{1}{4 A^{*} B^{*}} \frac{\mathrm{d} A^{*}}{\mathrm{~d} R_{K}^{*}}\left(\frac{1}{A^{*}} \frac{\mathrm{d} A^{*}}{\mathrm{~d} R_{K}^{*}}+\frac{1}{B^{*}} \frac{\mathrm{d} B^{*}}{\mathrm{~d} R_{K}^{*}}\right)-\frac{1}{R_{K}^{*} A^{*} B^{*}} \frac{\mathrm{d} A^{*}}{\mathrm{~d} R_{K}^{*}}=0, \\
\frac{1}{2 A^{*} B^{*}} \frac{\mathrm{d}^{2} A^{*}}{\mathrm{~d} R_{K}^{* 2}}-\frac{1}{4 A^{*} B^{*}} \frac{\mathrm{d} A^{*}}{\mathrm{~d} R_{K}^{*}}\left(\frac{1}{A^{*}} \frac{\mathrm{d} A^{*}}{\mathrm{~d} R_{K}^{*}}+\frac{1}{B^{*}} \frac{\mathrm{d} B^{*}}{\mathrm{~d} R_{K}^{*}}\right)-\frac{1}{R_{K}^{*} B^{* 2}} \frac{\mathrm{d} B^{*}}{\mathrm{~d} R_{K}^{*}}=0 .
\end{gathered}
$$

From the previous two equations we have:

$$
\begin{aligned}
& \frac{\mathrm{d} B^{*}}{B^{*}}=-\frac{\mathrm{d} A^{*}}{A^{*}}, \\
& B^{*}=\frac{K_{1}}{A^{*}} .
\end{aligned}
$$

The value of the constant $K_{1}$ can be deduced by imposing that, at infinity, the flat metric in Equation (8.6) must be recovered. In other terms, we must impose:

$$
\lim _{R_{K}^{*} \rightarrow \infty} A^{*}\left(R_{K}^{*}\right)=\lim _{R_{K}^{*} \rightarrow \infty} B^{*}\left(R_{K}^{*}\right)=1 .
$$

From Equation (8.32), taking into account the condition in Equation (8.33), we have:

$$
B^{*}=\frac{1}{A^{*}}
$$

The foregoing, taking into account Equation (8.11), we can be also written as follows:

$$
g_{00} g_{11}=-1 .
$$

Now, from Equations (8.24), (8.28) and (8.34), we have:

$$
\begin{gathered}
A^{*}+\frac{R_{K}^{*} A^{*}}{2}\left[\frac{1}{A^{*}} \frac{\mathrm{d} A^{*}}{\mathrm{~d} R_{K}^{*}}-A^{*} \frac{\mathrm{d}}{\mathrm{d} R_{K}^{*}}\left(\frac{1}{A^{*}}\right)\right]-1=0, \\
A^{*}+R_{K}^{*} \frac{\mathrm{d} A^{*}}{\mathrm{~d} R_{K}^{*}}-1=\frac{\mathrm{d}}{\mathrm{d} R_{K}^{*}}\left(R_{K}^{*} A^{*}\right)-1=0, \\
A^{*}=1+\frac{K_{2}}{R_{K}^{*}} .
\end{gathered}
$$

Finally, by setting $K_{2}=-K_{s}$ (just in order to further simplify the notation), from Equations (8.10), (8.34) and (8.38), we obtain: 


$$
\mathrm{d} s^{* 2}=\left(1-\frac{K_{s}}{R_{K}^{*}}\right) c^{2} \mathrm{~d} t^{* 2}-\frac{d R_{K}^{* 2}}{1-\frac{K_{s}}{R_{K}^{*}}}-R_{K}^{* 2}\left(\mathrm{~d} \theta^{2}+\sin ^{2} \theta \mathrm{d} \varphi^{2}\right) .
$$

The previous represents the Schwarzschild-Like Form for the Vacuum Solution.

The coordinate $R_{K}^{*}$, once again, does not necessarily coincide with the one derived in equation (7.46), basing on which the particular (initial) metric in Equation (8.6) has been built. On this subject, if we temporarily modify the notation by replacing $s^{*}$ with $s, t^{*}$ with $t, R_{K}^{*}$ with $R, K_{s}$ with $\alpha$, denoting with $r(r>0)$ the initial Flat Coordinate, which here does not coincide with the one defined in Equation (7.1), the Original Schwarzschild (Exact) Solution [7] is immediately obtained:

$$
\left\{\begin{array}{l}
\mathrm{d} s^{2}=\left(1-\frac{\alpha}{R}\right) c^{2} \mathrm{~d} t^{* 2}-\frac{\mathrm{d} R^{2}}{1-\frac{\alpha}{R}}-R^{2}\left(\mathrm{~d} \theta^{2}+\sin ^{2} \theta \mathrm{d} \varphi^{2}\right) \\
R=\left(r^{3}+\alpha^{3}\right)^{\frac{1}{3}}
\end{array}\right.
$$

For the sake of completeness, it is worth underlying how the value of the arbitrary constant $\alpha$ was never addressed by Schwarzschild himself [7].

Now, if we denote with $\psi$ the gravitational potential, for an arbitrary metric, as is well known, we must have:

$$
g_{00}=\left(1+\frac{\psi}{c^{2}}\right)^{2}
$$

Far from the source, we can resort to the so-called Weak Field Approximation:

$$
g_{00}=\left(1+\frac{\psi}{c^{2}}\right)^{2} \cong 1+2 \frac{\psi}{c^{2}} .
$$

Therefore, if we consider $\psi=\phi$, the latter having been defined in Equations (7.35), by virtue of Equations (7.47) and (8.42) we have:

$$
g_{00} \cong 1+2 \frac{\phi}{c^{2}}=1-\cos ^{2} \chi=1-\frac{K}{R_{K}^{*}} \text {. }
$$

By carrying out a banal comparison between Equation (8.38) and (8.43), we immediately obtain $K_{s}=K$. If we set $K=R_{s}$, taking into account Equation (6.1), from Equation (8.43) we immediately obtain:

$$
g_{00} \cong 1-\frac{R_{s}}{R^{*}}=1-\frac{2 G M_{t o t}}{c^{2} R^{*}} .
$$

From Equations (8.35) and (8.39), taking into account the previous, we obtain, far from the source, the underlying well-known form:

$$
\mathrm{d} s^{* 2}=\left(1-\frac{2 G M_{t o t}}{c^{2} R^{*}}\right) c^{2} \mathrm{~d} t^{* 2}-\frac{\mathrm{d} R^{* 2}}{1-\frac{2 G M_{t o t}}{c^{2} R^{*}}}-\mathrm{d} R^{* 2}\left(\mathrm{~d} \theta^{2}+\sin ^{2} \theta \mathrm{d} \varphi^{2}\right) .
$$




\subsection{Schwarzschild-Like Solution: Alternative Derivation}

In order to set a useful parallelism between the conventional derivation, examined in the previous paragraph, and the one we are about to carry out, we will exactly start from the approximated metric derived in Equation (8.1).

According to our model, the (parameterized) proper radius is not influenced by the singularity; on the contrary, the (parameterized) predicted radius undergoes a reduction.

Therefore, bearing in mind that we resort to the subscript " $g$ " every time we refer to a quantity measured after the creation of the singularity, taking into account Equations (7.43) and (7.53), from the metric in Equation (8.1) (no singularity) we obtain, in case of singularity, the following:

$$
\mathrm{d} s_{g}^{* 2}=c^{2} \mathrm{~d} t^{* 2}-\mathrm{d} R_{K}^{* 2}-R_{K}^{* 2} \sin ^{2} \chi\left(\mathrm{d} \theta^{2}+\sin ^{2} \theta \mathrm{d} \varphi^{2}\right) .
$$

By virtue of Equation (7.48), the foregoing can already acquire the following form:

$$
\mathrm{d} s_{g}^{* 2}=c^{2} \mathrm{~d} t^{* 2}-\mathrm{d} R_{K}^{* 2}-\left(1-\frac{K}{R_{K}^{*}}\right) R_{K}^{* 2}\left(\mathrm{~d} \theta^{2}+\sin ^{2} \theta \mathrm{d} \varphi^{2}\right) .
$$

The previous represents nothing but an (approximated) "analytic solution", built without taking into account the modified values of the Space and Time Quanta, related to each other as established by Equation (7.69). The abovementioned condition is clearly expressed by means of $g_{00}$, the value of which is manifestly unitary.

The metric in Equation (8.47) can be rewritten in the underlying form:

$$
\left.\mathrm{d} s_{g}^{* 2}\right|_{g_{00}=1}=c^{2} \mathrm{~d} t^{* 2}-\left.\mathrm{d} R_{p, g}^{* 2}\right|_{g_{00}=1}-\left.R_{c, g}^{* 2}\right|_{g_{00}=1}\left(\mathrm{~d} \theta^{2}+\sin ^{2} \theta \mathrm{d} \varphi^{2}\right) \text {. }
$$

In other terms, we have carried out the following positions:

$$
\begin{gathered}
\left.R_{c, g}^{*}\right|_{g_{00}=1}=R_{c}^{*} \sin \chi=R_{K}^{*} \sin \chi=R_{K}^{*} \sqrt{1-\frac{K}{R_{K}^{*}}}, \\
\left.\mathrm{~d} R_{p, g}^{*}\right|_{g_{00}=1}=\mathrm{d} R_{p}^{*}=\mathrm{d} R_{K}^{*} .
\end{gathered}
$$

Now, from Equations (7.69) and (7.70), we immediately obtain:

$$
\Delta t_{\min , g}^{*}(\chi)=\Delta t_{\min , \chi}^{*}=\frac{\Delta t_{\min , \pi / 2}^{*}}{\eta_{\Delta r_{\min }}^{*}}=\frac{\Delta t_{\min }^{*}}{\eta_{\Delta r_{\min }}^{*}}=\Delta t_{\min }^{*} \sqrt{1-\frac{K}{R_{K}^{*}}} .
$$

In the light of the previous, we can write:

$$
\mathrm{d} t_{g}^{*}=\frac{\mathrm{d} t_{\pi / 2}^{*}}{\eta_{\Delta r_{\min }}^{*}}=\frac{\mathrm{d} t^{*}}{\eta_{\Delta r_{\min }}^{*}}=\mathrm{d} t^{*} \sqrt{1-\frac{K}{R_{K}^{*}}}
$$

From Equation (8.49), taking into account Equations (7.59) and (7.67), we have:

$$
\left.R_{c, g}^{*}\right|_{g_{00}=1}=\mathcal{N} \Delta R_{\min }^{*} \sin \chi=\mathcal{N} \sin \chi \Delta r_{\min , \pi / 2}=\mathcal{N} \Delta r_{\min , \chi} .
$$

By resorting to Equations (7.59), (7.67), (8.3) and (8.49), we can temporarily 
introduce the two following Non-Dimensional(Normalized) Coordinates:

$$
\begin{gathered}
\bar{R}_{c}^{*}=\frac{R_{c}^{*}}{\Delta R_{\min }^{*}}=\frac{R_{K}^{*}}{\Delta r_{\min , \pi / 2}^{*}}=\frac{R_{K}^{*}}{\Delta R_{\min }^{*}}=\mathcal{N}, \\
\left.\bar{R}_{c, g}^{*}\right|_{g_{00}=1}=\frac{\left.\bar{R}_{c, g}^{*}\right|_{g_{00}=1}}{\Delta r_{\min , \chi}^{*}}=\frac{R_{K}^{*} \sin \chi}{\Delta r_{\min , \chi}^{*}}=\frac{R_{K}^{*}}{\Delta r_{\min , \pi / 2}^{*}}=\frac{R_{K}^{*}}{\Delta R_{\text {min }}^{*}}=\mathcal{N} .
\end{gathered}
$$

Evidently, the value of the (parameterized) predicted radius, as long as it is expressed in terms of Space-Quanta, can be regarded as being constant. In other terms, from Equation (8.54) and (8.55) we can banally write:

$$
\bar{R}_{c}^{*}=\left.\bar{R}_{c, g}^{*}\right|_{g_{00}=1} \text {. }
$$

If we want to take into account the quanta variability, we must replace $\mathrm{d} t^{*}$ with $\mathrm{d} t_{g}^{*}$. In other terms, we have to modify the value of $g_{00}$, so far still unitary.

Consequently, by virtue of Equation (8.52), we must impose:

$$
g_{00}=1-\frac{K}{R_{K}^{*}}=\frac{1}{\eta_{\Delta r_{\min }}^{* 2}} .
$$

Evidently, we have simply changed the Units of Measurement (we have modified the scale factor), nothing else: $g_{00}$, in fact, simply reveals how we measure time (and space).

By virtue of Equation (8.57), we can rewrite the metric in Equation (8.47) as follows:

$$
\begin{aligned}
\left.\mathrm{d} s_{g}^{* 2}\right|_{g_{00}=1 / \eta^{2}}= & \left(1-\frac{K}{R_{K}^{*}}\right) c^{2} \mathrm{~d} t^{* 2}-\left.\mathrm{d} R_{p, g}^{* 2}\right|_{g_{00}=1 / \eta^{2}} \\
& -\left.R_{c, g}^{* 2}\right|_{g_{00}=1 / \eta^{2}}\left(\mathrm{~d} \theta^{2}+\sin ^{2} \theta \mathrm{d} \varphi^{2}\right) .
\end{aligned}
$$

From Equations (8.49) and (8.50), with obvious meaning of the notation,

$$
\begin{gathered}
\left.R_{c, g}^{*}\right|_{g_{00}=1 / \eta^{2}}=\left.\eta_{\Delta r_{\text {min }}}^{*} R_{c, g}^{*}\right|_{g_{00}=1}=R_{K}^{*}, \\
\left.\mathrm{~d} R_{p}^{*}\right|_{g_{00}=1 / \eta^{2}}=\left.\eta_{\Delta r_{\text {min }}}^{*} \mathrm{~d} R_{p, g}^{*}\right|_{g_{00}=1}=\eta_{\Delta r_{\text {min }}} \mathrm{d} R_{K}^{*}=\frac{\mathrm{d} R_{K}^{*}}{\sqrt{1-\frac{K}{R_{K}^{*}}}} .
\end{gathered}
$$

Now, by substituting Equations (8.59) and (8.60) into Equation (8.58), we can finally write the so-called Droste Solution [46]:

$$
\mathrm{d} s_{g}^{* 2}=\left(1-\frac{K}{R_{K}^{*}}\right) c^{2} \mathrm{~d} t^{* 2}-\frac{\mathrm{d} R_{K}^{* 2}}{1-\frac{K}{R_{K}^{*}}}-R_{K}^{* 2}\left(\mathrm{~d} \theta^{2}+\sin ^{2} \theta \mathrm{d} \varphi^{2}\right) .
$$

Obviously, according to Equation (7.46), $R_{K}^{*}>0$ (since $\chi>0$ ).

For the sole purpose of simplifying the notation, the first member Equation (8.58) has been replaced with $\mathrm{d} s_{g}^{* 2}$ : this relabelling, at a first glance misleading, is clearly legitimised by the fact that, in Equation (8.61), the value of $g_{00}$ is ma- 
nifestly equal to the one defined in Equation (8.57).

It is worth noticing how, resorting to Equation (7.72), the metric in Equation (8.61) may immediately acquire the so-called Lenz-Schiff form [47]:

$$
\mathrm{d} s_{g}^{* 2}=\left[1-\left(\frac{v}{c}\right)^{2}\right] c^{2} \mathrm{~d} t^{* 2}-\frac{\mathrm{d} R_{K}^{* 2}}{1-\left(\frac{v}{c}\right)^{2}}-R_{K}^{* 2}\left(\mathrm{~d} \theta^{2}+\sin ^{2} \theta \mathrm{d} \varphi^{2}\right) .
$$

Now, from Equation (8.61), taking into account Equation (7.54), we obtain:

$$
\mathrm{d} s_{g}^{* 2}=\left(1-\frac{K}{x_{K}^{*}+K}\right) c^{2} \mathrm{~d} t^{* 2}-\frac{\mathrm{d} x_{K}^{* 2}}{1-\frac{K}{x_{K}^{*}+K}}-\left(x_{K}^{*}+K\right)^{2}\left(\mathrm{~d} \theta^{2}+\sin ^{2} \theta \mathrm{d} \varphi^{2}\right) .
$$

Clearly, by virtue of Equation (7.54), $x_{K}^{*}>0$. From the previous, we can immediately deduce the original form of the so-called Brillouin Solution [48]:

$$
\mathrm{d} s_{g}^{* 2}=\frac{c^{2} \mathrm{~d} t^{* 2}}{1+\frac{K}{x_{K}^{*}}}-\left(1+\frac{K}{x_{K}^{*}}\right) \mathrm{d} x_{K}^{* 2}-\left(x_{K}^{*}+K\right)^{2}\left(\mathrm{~d} \theta^{2}+\sin ^{2} \theta \mathrm{d} \varphi^{2}\right) .
$$

By virtue of Equation (8.60), as for the proper radius, we can now write:

$$
\begin{aligned}
& R_{p, g}^{*}=\int \frac{\mathrm{d} R_{K}^{*}}{\sqrt{1-\frac{K}{R_{K}^{*}}}}=\int \frac{\sqrt{\left(R_{K}^{*}-K\right)+K}}{\sqrt{R_{K}^{*}-K}} \mathrm{~d}\left(R_{K}^{*}-K\right) \\
& =2 \int \sqrt{\left(\sqrt{R_{K}^{*}-K}\right)^{2}+(\sqrt{K})^{2}} \mathrm{~d}\left(\sqrt{R_{K}^{*}-K}\right) \text {. }
\end{aligned}
$$

We have just found an integral of the following kind:

$$
\int \sqrt{y^{2}+(\sqrt{K})^{2}} \mathrm{~d} y=\frac{K}{2} \ln \left(y+\sqrt{y^{2}+K}\right)+\frac{y}{2} \sqrt{y^{\prime 2}+K}+C_{K} .
$$

Consequently, from the previous two equations we obtain:

$$
R_{p, g}^{*}=\int \frac{\mathrm{d} R_{K}^{*}}{\sqrt{1-\frac{K}{R_{K}^{*}}}}=\ln \left(\sqrt{R_{K}^{*}}+\sqrt{R_{K}^{*}-K}\right)+\sqrt{R_{K}^{*}\left(R_{K}^{*}-K\right)}+C_{K} .
$$

As for the constant $C_{K}$, by imposing $R_{p}^{*}(K)=0$, we immediately obtain:

$$
C_{K}=-K \ln \sqrt{K}
$$

Finally, from the previous two Equations we have:

$$
R_{p, g}=K \ln \left(\frac{\sqrt{R_{K}^{*}-K}+\sqrt{R_{K}^{*}}}{\sqrt{K}}\right)+\sqrt{R_{K}^{*}\left(R_{K}^{*}-K\right)} .
$$

The foregoing, by virtue of Equation (7.54), can be also written as follows:

$$
R_{p, g}=K \ln \left(\frac{\sqrt{R_{K}^{*}-K}+\sqrt{R_{K}^{*}}}{\sqrt{K}}\right)+\sqrt{R_{K}^{*}\left(R_{K}^{*}-K\right)} .
$$




\section{4. "Weak" Generalization and Real Schwarzschild form}

Taking into account Equations (7.46) and (7.54), we have:

$$
\lim _{\chi \rightarrow \pi / 2} \frac{x_{K}^{*}}{R_{K}^{*}}=\lim _{\chi \rightarrow \pi / 2} \frac{R_{K}^{*}-K}{R_{K}^{*}}=\lim _{\chi \rightarrow \pi / 2}\left(1-\frac{K}{R_{K}^{*}}\right)=1 .
$$

If we consider a real number $a$ (with $0<a<\infty$ ), from the foregoing we have:

$$
\lim _{\chi \rightarrow \pi / 2}\left(\frac{x_{K}^{*}}{R_{K}^{*}}\right)^{a}=\lim _{\chi \rightarrow \pi / 2} \frac{x_{K}^{* a}}{R_{K}^{* a}}=\lim _{\chi \rightarrow \pi / 2} \frac{x_{K}^{* a}+K^{a}}{R_{K}^{* a}}=1 .
$$

Therefore, far from the source, taking into account Equation (7.54), we obtain:

$$
\begin{gathered}
R_{K}^{* a} \cong x_{K}^{* a}+K^{a}=\left(R_{K}^{*}-K\right)^{a}+K^{a}, \\
R_{K}^{*} \cong\left[\left(R_{K}^{*}-K\right)^{a}+K^{a}\right]^{\frac{1}{a}}=\left(x_{K}^{* a}+K^{a}\right)^{\frac{1}{a}}=R_{K, a}^{*} .
\end{gathered}
$$

In the previous we have introduced a New Coordinate, denoted by $R_{K, a}^{*}$. Evidently, by virtue of Equation (7.54), we have $R_{K, a}^{*}=K$ at $\chi=0$. Moreover, it easy to verify how the derivative of $R_{K, a}^{*}$ with respect to $\chi \quad$ (with $0<\chi<\pi / 2$ ) is always positive. To summarise, taking into account Equations (7.49), (7.54), (8.72) and (8.74), we have:

$$
\begin{gathered}
R_{K, a}^{*}(0)=K, \\
\frac{\mathrm{d} R_{K, a}^{*}}{\mathrm{~d} \chi}=\left(x_{K}^{* a}+K^{a}\right)^{\frac{1-a}{a}} x_{K}^{* a-1} \frac{\mathrm{d} x_{K}^{*}}{\mathrm{~d} \chi}>0, \\
\lim _{\chi \rightarrow \pi / 2} \frac{R_{K, a}^{*}}{R_{K}^{*}}=\lim _{\chi \rightarrow \pi / 2}\left(\frac{x_{K}^{* a}+K^{a}}{R_{K}^{* a}}\right)^{\frac{1}{a}}=1 .
\end{gathered}
$$

Therefore, the new coordinate defined in Equation (8.74) and the one defined in Equation (7.46) are fully interchangeable (since they behave exactly the same way) [49].

In other terms, we have:

$$
R_{K, a}^{*} \cong R_{K}^{*}
$$

From Equation (8.74), by setting $a=3$, we obtain:

$$
R_{K, 3}^{*}=\sqrt[3]{x_{K}^{* 3}+K^{3}}
$$

By substituting the previous into Equation (8.61), we finally obtain the real Schwarzschild form (form and not exact solution, since $x_{K}^{*}$ does not coincide with the coordinate in the initial flat metric of the original derivation) [7].

\subsection{Gravitational Redshift: Approximated Derivation}

If we impose the conservation of energy, we can write, with obvious meaning of the notation, the following:

$$
E_{\text {photon }, \chi}=h_{\chi} f_{\chi}=h_{\pi / 2} f_{\pi / 2}=E_{\text {photon }, \pi / 2} .
$$


From the previous, by virtue of Equation (7.13), we obtain:

$$
\frac{h_{\chi}}{h_{\pi / 2}}=\frac{f_{\pi / 2}}{f_{\chi}}=\sin ^{2} \chi .
$$

If we assume the Speed of Light Constancy (actually, as we will see in the next paragraph, this assumption cannot be regarded as properly correct), we have:

$$
c=\lambda_{\chi} f_{\chi}=\lambda_{\pi / 2} f_{\pi / 2} \text {. }
$$

The two foregoing relations allows to define a New Scale Parameter, denoted by $\eta_{\lambda}$ :

$$
\frac{\lambda_{\pi / 2}}{\lambda_{\chi}}=\frac{h_{\pi / 2}}{h_{\chi}}=\frac{1}{\sin ^{2} \chi}=\eta_{\lambda} .
$$

According to the definition of Gravitational Redshift [3], usually denoted by $Z$, from the previous we have:

$$
z=\frac{\lambda_{\pi / 2}-\lambda_{\chi}}{\lambda_{\chi}}=\frac{\lambda_{\pi / 2}}{\lambda_{\chi}}-1=\eta_{\lambda}-1 .
$$

From Equations (7.10) and (7.68) we obtain:

$$
\lim _{\chi \rightarrow \pi / 2} \frac{\eta_{\Delta r_{\min }}}{\eta_{\lambda}}=\lim _{\chi \rightarrow \pi / 2} \frac{\eta_{\Delta r_{\min }}^{*}}{\eta_{\lambda}}=\lim _{\chi \rightarrow \pi / 2} \sin \chi=1 .
$$

Consequently, by virtue of the two previous Equations, far from the source we have:

$$
z \cong \eta_{\Delta r_{\min }}-1=\frac{1}{\sin \chi}-1 .
$$

From the foregoing, taking into account Equations (7.48) and (8.84), we obtain:

$$
z=\frac{\lambda_{\pi / 2}-\lambda_{\chi}}{\lambda_{\chi}}=\frac{\lambda_{\infty}-\lambda_{R^{*}}}{\lambda_{R^{*}}}=\frac{1}{\sqrt{1-\frac{K}{R_{K}^{*}}}}-1 .
$$

If we set $K=R_{s}$, by virtue of Equations (6.1), (7.29) and (7.46), the previous can be finally written in the underlying well-known form:

$$
z=\frac{1}{\sqrt{1-\frac{2 G M_{t o t}}{c^{2} R^{*}}}}-1
$$

\section{9. "Black Holes": A Mystery Still Unsolved}

On the 10th of April 2019, the world was allowed to see something defined as the first ever "image" of the alleged (super massive) Black Hole placed in the heart of M87, a (super) giant elliptical galaxy in the constellation Virgo [50] [51]. The image, captured by the Event Horizon Telescope (EHT, an "earth-size" telescope actually consisting of a global network of radio telescopes), shows an object that, despite its undeniable compatibility with a so-called Kerr Black Hole [52], does 
not necessarily represent a Black Hole.

The "picture", in fact, lends itself to several interpretations, some of which even based on GR. On this subject, it is worth underlining how Einstein himself, who was probably never sure that his field equations would ever be exactly solved, limited himself to recognizing the mathematic validity of the elegant (exact) solution found by Schwarzschild [7]. To be clearer, beyond any doubt, Einstein actually never "dreamt" of the existence of astronomical objects provided with the features of a Black Hole.

\subsection{An Alternative (Relativistic) Point of View: ECOs (Short Account)}

Amongst the alternative interpretations of the image captured by the EHT, one cannot help but mention the one based on the existence of the so-called Eternally Collapsing Objects (ECOs), some sort of self-gravitating (and constantly contracting) extremely hot "plasmoids" coherent with GR [53] [54] [55]. These objects, despite their compatibility with GR (or, paradoxically, exactly by virtue of their compatibility with GR, at least in its original conception), are anything but related to the existence of Black Holes (and, consequently, of Event Horizons and Trapped Surfaces) [56]. In ECOs, very briefly, the collapse may occur with a local speed close to that of light: therefore, the accretion process may generate a very small (although not null, unlike a "real" Black Hole) radiation output, which may be erroneously interpreted as related to the presence of an Event Horizon.

For this reason, ECOs may appear extremely similar to the object recently captured by the EHT, hastily regarded as the ultimate evidence of the existence of Black Holes.

\subsection{Beyond the Metrics: Velocity as a Complex Vector (Short Account)}

We have so far discussed in terms of warped space (due to a singularity): actually, if it were so, we would be forced to admit that, in a certain sense, the shape of the Universe could be modified with respect to something else, taken as a reference. Alternatively, we can imagine that, although the space value undergoes local modifications, the Universe may preserve its original shape (a 4-Ball, according to the model herein proposed).

Therefore, the speed, the magnitude of which is defined by Equation (7.38), may consist of a real component, defined by Equation (7.41), and of an imaginary one, defined by Equation (7.42), exclusively related to the variability of the Planck Constant.

In other terms, denoting with $v^{*}$ the Complex Velocity (with $\left|v^{*}\right|=v$ ), from Equations (7.38), (7.41) and (7.42), we can write:

$$
v^{*}=v_{R e}^{*}+i v_{I m}^{*}=c \cos \chi=c \sqrt{\frac{R_{s}}{R^{*}}},
$$




$$
\begin{gathered}
v_{R e}^{*}=v_{I}=v \sin \chi=c \sin \chi \cos \chi=c \sqrt{\left(1-\frac{R_{s}}{R^{*}}\right) \frac{R_{s}}{R^{*}}}, \\
v_{I m}^{*}=v_{I I}=v \cos \chi=c \cos ^{2} \chi=c \frac{R_{s}}{R^{*}} .
\end{gathered}
$$

It is easy to verify how $v_{R e}^{*}$ reaches a maximum at $R^{*}=2 R_{s}$ (when $\chi=\pi / 4$ ): consequently, there may concretely be a point in correspondence of which, as counterintuitive as the phenomenon may appear, a free falling particle starts to slow down.

Light would not be an exception to the rule. Taking into account Equations (7.32) and (7.33), we can write, with obvious meaning of the notation, the following:

$$
\begin{gathered}
c^{*}=c_{R e}^{*}+i c_{I m}^{*}, \\
c_{R e}^{*}=c \sin \chi=c \sqrt{\left(1-\frac{R_{s}}{R^{*}}\right)}, \\
c_{I m}^{*}=c \cos \chi=c \sqrt{\frac{R_{s}}{R^{*}}} .
\end{gathered}
$$

Therefore, light may concretely (and monotonically) slow down in approaching the "singularity": part of its energy, in fact, may compensate for the reduction of the space-quanta value.

In relativistic terms [3], taking into account Equation (8.52) (with $K=R_{s}$ and, consequently, $R_{K}^{*}=R^{*}$ ), denoting with $l_{R e}^{*}$ the (proper) distance actually covered (the motion is considered as being purely radial), from Equations (9.2) and (9.5) we obtain:

$$
\begin{gathered}
v^{\prime}=\frac{\mathrm{d} l_{R e}^{*}}{\mathrm{~d} t^{*}}=\frac{\mathrm{d} l_{R e}^{*}}{\mathrm{~d} t_{g}^{*}} \frac{\mathrm{d} t_{g}^{*}}{\mathrm{~d} t^{*}}=v_{R e}^{*} \sqrt{1-\frac{R_{s}}{R^{*}}}=c\left(1-\frac{R_{s}}{R^{*}}\right) \sqrt{\frac{R_{s}}{R^{*}}}, \\
c^{\prime}=\frac{\mathrm{d} l_{R e, \text { Light }}^{*}}{\mathrm{~d} t^{*}}=\frac{\mathrm{d} l_{R e, \text { Light }}^{*}}{\mathrm{~d} t_{g}^{*}} \frac{\mathrm{d} t_{g}^{*}}{\mathrm{~d} t^{*}}=c_{R e}^{*} \sqrt{1-\frac{R_{s}}{R^{*}}}=c\left(1-\frac{R_{s}}{R^{*}}\right) .
\end{gathered}
$$

In the light of what we have deduced, we could even imagine that, at the time referring to the image captured by the EHT, light had not yet reached the (super massive) object in question: according to this hypothesis, we will be able to see, with the passing of time, all the astronomical objects currently regarded as Black Holes.

\section{Final Remarks and Conclusions}

In spite of its length, this paper represents nothing but a mere introduction to a work considerably wider and still in progress: for the sake of brevity, therefore, several aspects, which surely deserve more accurate discussion, are merely touched upon.

We postulate the existence of a further spatial dimension, not directly perceivable. 
The Universe is identified with a 4-Ball involved in an apparent cyclic evolution.

The concept of material point is replaced by the one of material segment: in detail, what is perceived as being a point may actually be a segment crossing the centre of the 4-Ball [6] [12] [13] [18]. Two antipodal points, since they evidently represent the endpoints of the same segment, must be considered as being a unique entity: in other terms, the Universe may be characterized by a (Global) Central Symmetry [6] [12] [13] [18].

Time and space are considered as being quantized physical quantities.

The extra spatial dimension and the space quantization allow the writing of all the relativistic equations concerning energy, although with different connotations [6] [12] [18]. The conservation of energy is derived by considering an additional non-material component [6] [12], related to the concept of Quantum Potential [14] [15]. In particular, albeit the punctual mass is considered as being constant, the linear mass (the four-dimensional mass) may undergo a reduction with the increasing of speed [6] [12]. The non-material component, very intuitively, may simply compensate for the linear mass reduction.

Although the topic, for the sake of brevity, is herein not addressed, it is worth specifying how, according to our model, the centre of the 4-Ball with which we identify the Universe cannot be exactly located. The quantization herein proposed must be regarded as approximate. Actually, the "centre" may consist in a minimal 4-Ball, characterized by a diameter equal to the minimal length derived by resorting to the Generalised Uncertainty Principle [17]: inside the corresponding border (a minimal hyper-sphere), the concept of "separation" may become de facto meaningless.

Relativity, at least in its original formulation, is clearly a $3 D+1$ theory [3] [4], while the Universe herein hypothesized is evidently based on a $4 D+1$ model: consequently, a direct comparison turns out to be de facto impossible. In our theory, however, the Universe is considered as being "triune" (one in three): in other terms, its features are deduced by resorting to the superposition of three $3 D+1$ sub-models, so bypassing the obstacle [6] [12] [18].

Time is considered as being absolute, although instruments and devices of whatever kind, commonly employed to measure it, may be significantly influenced by motion and gravity. To be clearer, time does not slow down with the increasing of speed or in approaching a gravitational source [6] [12] [13] [18].

Apparently, muons succeed in covering a distance not compatible with their mean lifetime: actually, they may simply exploit a shortcut, as it were. According to our model, in fact, the proper distance between whatever couple of points depends on their speed and, what is more, is no longer symmetric. In other terms, the very moment a particle starts moving, all the proper distances must be redefined as a function of the speed: on the contrary, all the angular distances preserve their initial value. Obviously, both the angular distances and the proper ones vary during the motion. This scenario is formally coherent with the Lorentz 
Transformations [3] [4] [16] [19], the meaning of which, however, is completely altered. Amongst the significant consequences that arise from our approach, the possibility of (apparently) moving faster than light stands out [13].

The Planck Constant is downgraded to the rank of parameter.

As it is well known, the possibility of a variable Planck "Constant", both in time [6] [30] [31] [32] and in space [18] [41] [42] [43] [44], represents anything but a novelty. According to the theory herein expounded, a time dependent Planck Constant may represent the real cause of the so-called Cosmological Redshift [3], no longer ascribable to a concrete variation of cosmological distances. [6] In particular, we have shown how an opportune space-quantization [17] [18] allows a rewriting of the first Friedmann-Lemaitre Equation [2] as a function of a variable Planck Constant. The quantization may also allow to bypass the singularity at $t=0$ by means of a "bounce" [8] [9] [10], herein not addressed. To extremely simplify, the time at which the Planck Constant should mathematically assume a null value would fall between two consecutive timesteps.

The local variability of the Planck Constant may also cause the so-called Gravitational Redshift: in passing through points characterized by different values of the gravitational potential, light undeniably modifies its frequency [3], but time is not involved in the phenomenon [18]. A cyclic behavior (with a period approximately equal to one year) in the decay rates of several radionuclides may lend support to the existence of a connection between the ellipticity of Earth's Orbit and the variability of the Planck Constant [44].

The atomic clocks' "ticking" is governed by the Fine Structure Constant, which may lose its constancy (both in time and in space) by virtue of its dependence on $h$ [57] [58] [59] [60] [61]. According to our model, even though the atomic clocks are undoubtedly influenced by gravity, time may keep on flowing undisturbed.

The existence of Black Holes is brought into question.

A Schwarzschild-Like solution [7] [46] [48] can be derived without resorting to GR. According to GR, a particle may cross the Event Horizon and continue on its way towards the Singularity: however, an external observer (ideally placed at infinity), may eternally see the particle lying on the Event Horizon, as if it were frozen [3] [4]. According to our model, an Event Horizon may not even exist, even if the entire mass of the Universe were concentrated in a single point: in this case, however, the speed of a free-falling particle may reach a maximum at $R^{*}=2 R_{s}$, becoming null at $R^{*}=R_{s}$. (i.e. when it reaches the "singularity"). Light, in travelling towards the "singularity", may progressively slow down, stopping at $R^{*}=R_{s}$ : consequently, what we are used to identifying as an Event Horizon could actually be nothing but a region not yet reached by light (at the time referring to the image we are allowed to observe). Such a scenario simply arises from considering velocity as a complex vector: in approaching a gravitational source, the magnitude (the modulus) of the velocity of a free-falling par- 
ticle keeps on increasing, while the magnitude of the velocity of light is constantly equal to $c$.

\section{Acknowledgements}

This paper is dedicated to my father, Antonio Cataldo, who unexpectedly passed away on the 11th of June 2016 .

\section{Conflicts of Interest}

The author declares no conflicts of interest regarding the publication of this paper.

\section{References}

[1] Harrison, E.R. (1967) Classification of Uniform Cosmological Models. Monthly Notices of the Royal Astronomical Society, 137, 69-79.

https://doi.org/10.1093/mnras/137.1.69

[2] Friedmann, A. (1922) Über die Krümmung des Raumes. Zeitschrift für Physik, 10, 377-386. https://doi.org/10.1023/A:1026751225741

[3] Cheng, T.-P. (2005) Relativity, Gravitation and Cosmology: A Basic Introduction. Oxford University Press Inc., New York.

[4] Einstein, A. (1916) Relativity: The Special and General Theory (Translated by Lawson, R.W., 1920). H. Holt and Co., New York. https://archive.org/details/cu31924011804774 https://doi.org/10.4324/9780203198711

[5] Zeldovich, Y. (1961) The Equation of State at Ultrahigh Densities and Its Relativistic Limitations. Journal of Experimental and Theoretical Physics, 41, 1609-1615. http://www.jetp.ac.ru/cgi-bin/dn/e_014_05_1143.pdf

[6] Cataldo, C. (2019) Towards a New Concept of Closed System: From the Oscillating Universe to the EM-Drive. International Journal of Advanced Engineering Research and Science, 6, 43-52. https://doi.org/10.22161/ijaers.6.2.7

[7] Schwarzschild, K. (1916) Über das Gravitationsfeld eines Massenpunktes nach der Einsteinschen Theorie. Sitzungsber. der Deutschen Akad. der Wiss. zu Berlin, 189196. http://zelmanov.ptep-online.com/papers/zj-2008-03.pdf

[8] Turok, N. and Steinhardt, P. (2005) Beyond Inflation: A Cyclic Universe Scenario. Physica Scripta, T117, 76-85. https://arxiv.org/abs/hep-th/0403020 https://doi.org/10.1238/Physica.Topical.117a00076

[9] Gielen, S. and Turok, N. (2016) Perfect Quantum Cosmological Bounce. Physical Review Letters, 117, Article ID: 021301. https://doi.org/10.1103/PhysRevLett.117.021301

[10] Ijjas, A. and Steinhardt, P.J. (2016) Classically Stable Nonsingular Cosmological Bounces. Physical Review Letters, 117, Article ID: 121304. https://doi.org/10.1103/PhysRevLett.117.121304

[11] Hubble, E.P. (1929) A Relation between Distance and Radial Velocity among Extra-Galactic Nebulae. Proceedings of the National Academy of Sciences of the United States of America, 15, 168-173. https://doi.org/10.1073/pnas.15.3.168

[12] Cataldo, C. (2016) From the Oscillating Universe to Relativistic Energy: A Review. Journal of High Energy Physics, Gravitation and Cosmology, 3, 68-77. 
https://doi.org/10.4236/jhepgc.2017.31010

[13] Cataldo, C. (2016) Faster than Light: Again on the Lorentz Transformations. Applied Physics Research, 8, 17-24. https://doi.org/10.5539/apr.v8n6p17

[14] Bohm, D. (1952) A Suggested Interpretation of the Quantum Theory in Terms of "Hidden Variables" I. Physical Review, 85, 166-179. https://doi.org/10.1103/PhysRev.85.166

[15] Bohm, D. (1952) A Suggested Interpretation of the Quantum Theory in Terms of “Hidden Variables", II. Physical Review, 85, 180-193. https://doi.org/10.1103/PhysRev.85.180

[16] Lorentz, H.A. (1904) Electromagnetic Phenomena in a System Moving with Any Velocity Smaller than That of Light. Proceedings of the Royal Netherlands Academy of Arts and Sciences, 6, 809-831. http://www.dwc.knaw.nl/DL/publications/PU00014148.pdf

[17] Shalit-Margolyn, A. (2018) Minimal Quantities and Primary Measurable Variant of Gravity II. Strong Principle of Equivalence and Transition to High Energies. Advanced Studies in Theoretical Physics, 12, 79-103. https://doi.org/10.12988/astp.2018.823

[18] Cataldo, C. (2019) Black Holes: A Different Perspective. International Journal of Advanced Engineering Research and Science, 6, 119-131. https://doi.org/10.22161/ijaers.6.4.14

[19] Lorentz, H.A. (1909) The Theory of Electrons and Its Applications to the Phenomena of Light and Radiant Heat. B.G. Teubner, Leipzig, G. E. Stechert \& Co., New York. https://archive.org/details/electronstheory00lorerich

[20] Voigt, W. (1887) Ueber das Doppler'sche Princip. Nachrichten von der Königl. Gesellschaft der Wissenschaften und der G.-Aug.-Universität zu Göttingen, 2, 41-51. http://www.digizeitschriften.de/dms/img/?PPN=PPN252457072_1887\&DMDID=d $\underline{\mathrm{md} \log 12}$

[21] Maxwell, J.C. (1873) A Treatise on Electricity and Magnetism. Clarendon Press, Oxford. https://archive.org/details/electricandmagne01maxwrich

[22] Di Mauro, P. and Notarrigo, S. (1997) Sull'Invarianza delle Equazioni di Maxwell. Atti del XVI Congresso Nazionale di Storia della Fisica e delP Astronomia, 355-360. http://www.lascuolaitalica.it/pubbl5.htm

[23] Ghosal, S.K., Nandi, K.K. and Chakraborty, P. (1991) Passage from Einsteinian to Galilean Relativity and Clock Synchrony. Zeitschrift für Naturforschung A, 46, 256-258. https://doi.org/10.1515/zna-1991-0307

[24] Di Mauro, P. and Notarrigo, S. (1995) Critica delle usuali derivazioni delle trasformazioni di Lorentz. Comunicazione al LXXXI Congresso Nazionale SIF, Perugia, 4 Ottobre 1995. http://www.lascuolaitalica.it/pubbl12.htm

[25] Young, T. (1805) An Essay on the Cohesion of Fluids. Philosophical Transactions of the Royal Society of London, 95, 65-87. https://doi.org/10.1098/rstl.1805.0005

[26] Hubble, E.P. (1947) The 200-Inch Telescope and Some Problems It May Solve. Publications of the Astronomical Society of the Pacific, 59, 153-167.

https://doi.org/10.1086/125931

[27] Zwicky, F. (1929) On the Red Shift of Spectral Lines through Interstellar Space. Proceeding of the National Academy of Science of the United States of America, 15, 773-779. https://doi.org/10.1073/pnas.15.10.773

[28] Geller, M.J. and Peebles, P.J.E. (1972) Test of the Expanding Universe Postulate. The Astrophysical Journal, 174, 1-5. https://doi.org/10.1086/151462 
[29] LaViolette, P. (1986) Is the Universe Really Expanding? The Astrophysical Journal, 301, 544-553. https://doi.org/10.1086/163922

[30] Seshavatharam, U.V.S. and Lakshminarayana, S. (2013) Is Planck's Constant-A Cosmological Variable? International Journal of Astronomy, 2, 11-15. http://article.sapub.org/10.5923.j.astronomy.20130201.02.html

[31] Seshavatharam, U.V.S., Lakshminarayana, S. and Sai, B.V.S.T. (2013) Inadequacy of Modern Cosmology and Basics of Atomic Cosmology. http://www.rxiv.org/pdf/1303.0214v1.pdf

[32] Mangano, G., Fedele Lizzi, F. and Porzio, A. (2015) Inconstant Planck's Constant. International Journal of Modern Physics A, 30, Article ID: 1550209. https://doi.org/10.1142/S0217751X15502097

[33] Veneziano, G. (1986) A Stringy Nature Needs Just Two Constants. Europhysics Letters, 2, 199. https://doi.org/10.1209/0295-5075/2/3/006

[34] Adler, R.J. and Santiago, D.I. (1999) On Gravity and the Uncertainty Principle. Modern Physics Letters A, 14, 1371-1381. https://doi.org/10.1142/S0217732399001462

[35] Maggiore, M. (1994) Black Hole Complementarity and the Physical Origin of the Stretched Horizon. Physical Review D, 49, 2918-2921. https://doi.org/10.1103/PhysRevD.49.2918

[36] Capozziello, S., Lambiase, G. and Scarpetta, G. (2000) Generalized Uncertainty Principle from Quantum Geometry. International Journal of Theoretical Physics, 39, 15-22. https://doi.org/10.1023/A:1003634814685

[37] Wong, K.C., Suyu, S.H., Auger, M.W., Bonvin, V., Courbin, F., et al. (2016) H0LiCOW IV. Lens Mass Model of HE 0435-1223 and Blind Measurement of Its Time-Delay Distance for Cosmology. Monthly Notices of the Royal Astronomical Society, 465, 4895-4913. https://doi.org/10.1093/mnras/stw3077

[38] Bonvin, V., Courbin, F., Suyu, S.H., Marshall, P.J., Rusu, C., et al. (2016) H0LiCOW V. New COSMOGRAIL Time Delays of HE 0435-1223: H0 to 3.8 Percent Precision from Strong Lensing in a Flat $\Lambda$ CDM Model. Monthly Notices of the Royal Astronomical Society, 465, 4914-4930. https://doi.org/10.1093/mnras/stw3006

[39] Sarkar, S., Guffanti, A., et al. (2016) Marginal Evidence for Cosmic Acceleration from Type Ia Supernovae. Scientific Reports, 6, Article No. 35596. https://doi.org/10.1038/srep35596

[40] Hamilton, A.J.S. and Lisle, J.P. (2008) The River Model of Black Holes. American Journal of Physics, 76, 519-532. https://doi.org/10.1119/1.2830526

[41] Kentosh, J. and Mohageg, M. (2012) Global Positioning System Test of the Local Position Invariance of Planck's Constant. Physical Review Letters, 108, Article ID: 110801. https://doi.org/10.1103/PhysRevLett.108.110801

[42] Berengut, J.C. and Flambaum, V.V. (2012) Comment on "Global Positioning System Test of the Local Position Invariance of Planck's Constant”. Physical Review Letters, 109, Article ID: 068901. https://doi.org/10.1103/PhysRevLett.109.068901

[43] Kentosh, J. and Mohageg, M. (2012) Reply to Comment on "Global Positioning System Test of the Local Position Invariance of Planck's Constant”. Physical Review Letters, 109, Article ID: 068902. https://doi.org/10.1103/PhysRevLett.109.068902

[44] Hutchin, R.A. (2016) Experimental Evidence for Variability in Planck's Constant. Optics and Photonics Journal, 6, 124-137. https://doi.org/10.4236/opj.2016.66015

[45] Antoci, S. (2003) David Hilbert and the Origin of the "Schwarzschild Solution". https://arxiv.org/abs/physics/0310104 
[46] Droste, J. (1917) The Field of a Single Centre in Einstein's Theory of Gravitation and the Motion of a Particle in That Field. Proceedings of Royal Netherlands Academy of Arts and Sciences, 19I, Amsterdam, 197-215.

http://www.dwc.knaw.nl/DL/publications/PU00012346.pdf

[47] Schiff, L.I. (1960) On Experimental Tests of the General Theory of Relativity. American Journal of Physics, 28, 340. https://doi.org/10.1119/1.1935800

[48] Brillouin, M. (1923) Les points singuliers de l'univers d'Einstein. Journal de Physique et le Radium, 4, 43-48. https://arxiv.org/pdf/physics/0002009.pdf https://doi.org/10.1051/jphysrad:019230040104300

[49] Abrams, L.S. (1989) Black Holes: The Legacy of Hilbert's Error. Canadian Journal of Physics, 67, 919-926. https://doi.org/10.1139/p89-158

[50] The Event Horizon Telescope Collaboration, et al. (2019) First M87 Event Horizon Telescope Results. I. The Shadow of the Supermassive Black Hole. The Astrophysical Journal Letters, 875, L1. https://doi.org/10.3847/2041-8213/ab0ec7

[51] The Event Horizon Telescope Collaboration, et al. (2019) First M87 Event Horizon Telescope Results. IV. Imaging the Central Supermassive Black Hole. The Astrophysical Journal Letters, 875, L4. https://doi.org/10.3847/2041-8213/ab0e85

[52] Kerr, R.P. (1963) Gravitational Field of a Spinning Mass as an Example of Algebraically Special Metrics. Physical Review Letters, 11, 237. https://doi.org/10.1103/PhysRevLett.11.237

[53] Mitra, A. (2006) Radiation Pressure Supported Stars in Einstein Gravity: Eternally Collapsing Objects. Monthly Notices of the Royal Astronomical Society, 369, 492-496. https://doi.org/10.1111/j.1365-2966.2006.10332.x

[54] Mitra, A. (2006) Sources of Stellar Energy, Einstein-Eddington Timescale of Gravitational Contraction and Eternally Collapsing Objects. New Astronomy, 12, 146-160. https://doi.org/10.1016/j.newast.2006.08.001

[55] Mitra, A. and Glendenning, N.K. (2010) Likely Formation of General Relativistic Radiation Pressure Supported Stars or "Eternally Collapsing Objects". Monthly Notices of the Royal Astronomical Society: Letters, 404, L50-L54. https://doi.org/10.1111/j.1745-3933.2010.00833.x

[56] Mitra, A. (2000) Non-Occurrence of Trapped Surfaces and Black Holes in Spherical Gravitational Collapse. Foundations of Physics Letters, 13, 543. https://doi.org/10.1023/A:1007810414531

[57] Webb, J.K., Flambaum, V.V., Churchill, C.W., Drinkwater, M.J. and Barrow, J.D. (1999) Search for Time Variation of the Fine Structure Constant. Physical Review Letters, 82, 884. https://doi.org/10.1103/PhysRevLett.82.884

[58] Webb, J.K., Murphy, M.T., Flambaum, V.V., et al. (2001) Further Evidence for Cosmological Evolution of the Fine Structure Constant. Physical Review Letters, 87, Article ID: 091301. https://doi.org/10.1103/PhysRevLett.87.091301

[59] Murphy, M.T., Webb, J.K. and Flambaum, V.V. (2003) Further Evidence for a Variable Fine-Structure Constant from Keck/HIRES QSO Absorption Spectra. Monthly Notices of the Royal Astronomical Society, 345, 609-638. https://doi.org/10.1046/j.1365-8711.2003.06970.x

[60] Prestage, J.D., Tjoelker, R.L. and Maleki, L. (1995) Atomic Clocks and Variations of the Fine Structure Constant. Physical Review Letters, 74, 3511-3514. https://doi.org/10.1103/PhysRevLett.74.3511

[61] Dzuba, V.A. and Flambaum, V.V. (2000) Atomic Optical Clocks and Search for Variation of the Fine-Structure Constant. Physical Review A, 61, Article ID: 034502. https://doi.org/10.1103/PhysRevA.61.034502 